Technische

Universität

Berlin

\author{
Robert A. Skutnik, Immanuel S. Geier, Martin Schoen
}

\title{
A biaxial nematic liquid crystal composed of matchbox-symmetric molecules
}

Open Access via institutional repository of Technische Universität Berlin

\section{Document type}

Journal article | Accepted version

(i. e. final author-created version that incorporates referee comments and is the version accepted for publication; also known as: Author's Accepted Manuscript (AAM), Final Draft, Postprint)

This version is available at

https://doi.org/10.14279/depositonce-14849

\section{Citation details}

Robert A. Skutnik, Immanuel S. Geier \& Martin Schoen (2020) A biaxial nematic liquid crystal composed of matchbox-symmetric molecules, Molecular Physics, 118:9-10,

https://doi.org/10.1080/00268976.2020.1726520.

This is an Accepted Manuscript of an article published by Taylor \& Francis in Molecular Physics on 11 Feb 2020, available online: http://www.tandfonline.com/10.1080/00268976.2020.1726520.

\section{Terms of use}

This work is protected by copyright and/or related rights. You are free to use this work in any way permitted by the copyright and related rights legislation that applies to your usage. For other uses, you must obtain permission from the rights-holder(s). 


\title{
A biaxial nematic liquid crystal composed of matchbox-symmetric molecules
}

\author{
Robert A. Skutnik ${ }^{\mathrm{a}}$, Immanuel S. Geier ${ }^{\mathrm{a}}$, and Martin Schoen ${ }^{\mathrm{a}, \mathrm{b}}$ \\ ${ }^{a}$ Stranski-Laboratorium für Physikalische und Theoretische Chemie, Technische Universität \\ Berlin, Straße des 17. Juni 115, 10623 Berlin, Germany; ${ }^{b}$ Department of Chemical \\ Engineering, Imperial College London, South Kensington Campus, London SW7 2AZ, \\ United Kingdom
}

\section{ARTICLE HISTORY}

Compiled January 30, 2020

\begin{abstract}
By means of Monte Carlo simulations in the isothermal-isobaric ensemble we investigate the structure and phase behaviour of a thermotropic liquid crystal composed of matchbox-symmetric (or board-like) molecules. Besides the isotropic phase the liquid crystal exhibits also uniaxial and biaxial nematic phases. The interaction potential is derived through an expansion in terms of Stone's rotational invariants [A. J. Stone, Mol. Phys. 78, 241-256, (1978)] that can be reexpressed in terms of Cartesian tensors. This latter formulation is particularly well suited for computer simulations. We analyse the orientation distribution function which allows us to distinguish between intrinsic and extrinsic biaxiality. In addition, we study the orientation-dependent correlation functions. In the limit of large intermolecular separations the value of the orientation correlation function corresponds to the uniaxial and biaxial order parameters which are coupled in a complex fashion.
\end{abstract}

\section{KEYWORDS}

Liquid crystal; nematic phase; biaxiality; Monte Carlo simulation

\section{Introduction}

Liquid crystals are a fascinating class of soft-matter systems capable of forming a host of ordered phases [1]. They consist of organic molecules (i.e., mesogens) where shape and flexibility of the molecular skeleton in conjunction with favourable thermodynamic conditions promote the formation of these ordered phases. The simplest one is the socalled nematic phase in which the distribution of the centres-of-mass of the mesogens is always isotropic. Yet, the mesogens align to a certain extent with a distinguished direction, the so-called nematic director $\widehat{\boldsymbol{n}}$. The nematic phase obviously has uniaxial symmetry in this case. However, this is not necessarily always so.

For example, in 1970 Freiser [2] and a little later Alben [3] hypothesised that it might be possible for nematic phases to exhibit biaxial instead of uniaxial symmetry. This conjecture rests upon a mean-field approach in both cases. In order for a nematic phase to exhibit biaxial symmetry, a second symmetry axis besides $\widehat{\boldsymbol{n}}$ needs to exist. In the realm of modern display technology, biaxial nematic liquid-crystalline materials 
are very interesting because of their short response times to applied external fields [4].

Usually, one distinguishes between intrinsic and extrinsic biaxiality [5]. Biaxiality is intrinsic if the mesogens themselves possess two symmetry axes. On the contrary, biaxiality is extrinsic if uniaxially symmetric mesogens are manipulated by external agents so that the system as a whole can exhibit a second symmetry axis under favourable conditions [6]. The external agent could be a second uniaxial compound in a binary mixture where the mesogens pertaining to different components prefer a T-shaped arrangement [7]. The same philosophy has been chosen in the work by Cuetos et al. [8]. However, unlike Ref. [7], where orientation-dependent Lennard-Jones-type of potentials were employed, Cuetos et al. base their study on (discontinuous) square-well interactions.

The first experimental realisation of a (lyotropic) biaxial nematic liquid crystal was reported by $\mathrm{Yu}$ and Saupe [9]. They studied a ternary mixture of potassium-laureate1-decanol and water. Another lyotropic liquid crystal that exhibits biaxial nematic phases is a ternary mixture of soap, detergent, and water studied by Oliveira et al. [10]. Lyotropic liquid crystals exhibiting extrinsic biaxiality have been studied by Stroobants and Lekkerkerker [11] who investigated a mixture of rod- and platelet-like mesogens within the framework of Onsager's theory. Using again Onsager's theory, Wensink et al. [12] report a first-order phase transition during which a stable biaxial nematic forms in a lyotropic liquid crystal.

As far as nematic phases with intrinsic biaxiality are concerned quite a bit of progress has been made because of advances in chemical synthesis. There are essentially two main molecular geometries that have been studied, namely bent-core $[13,14]$ and boardor matchbox-symmetric mesogens $[15,16]$. Both molecular architectures differ in the number of reflection planes. Whereas there are two such planes for bent-core structures, matchbox-shaped mesogens possess three of those. Slightly more extravagant architectures are those of organosiloxane tetrapodes [17] where matchbox-shaped central units are connected to a silicon atom. Because of this structure even biaxial short-range order could be detected by means of deuterium nuclear magnetic resonance (NMR). In a few cases even biaxial smectic phases have been reported [18, 19].

On the theoretical side quite a bit of work has already been devoted to biaxial nematic phases in liquid crystals. Tjipto-Margo and Evans [20] employed Onsager's theory to investigate the formation of uniaxial nematic phases in a liquid crystal composed of biaxial mesogens. At its core, Onsager's theory requires a calculation of the excluded volume of hard bodies. This problem has been investigated in depth by Mulder [21].

By means of computer simulations Allen [22] and later Camp and Allen [23] investigated the phase behaviour of intrinsically biaxial mesogens with only hard repulsive interactions. They proposed a set of order parameters also calculated in this work. These order parameters are the same as those employed also in the work by Mulder [21] and Straley [24].

Berardi et al. $[25,26]$ use a properly modified Gay-Berne potential in their simulations of a liquid crystal with intrinsic biaxiality. In a lattice Monte Carlo (MC) study Biscarini et al. [27] used an orientation dependence of the interaction potential based upon symmetry-adapted Wigner rotation matrices. Their model reduces to the Lebwohl-Lasher model in the limit of vanishing biaxiality. Results from mean-field theory are in good qualitative agreement with their MC data.

Recently, Cuetos et a. [28] employed MC simulations of board-like particles. These particles interact through purely entropic forces. The authors focus on the impact of the variation of the width-to-length ratio while keeping the thickness of the mesogens fixed. They found a rich phase behaviour including biaxial columnar and smectic phases. They 
also use Onsager's theory to predict the uniaxial-biaxial nematic phase transition.

A lattice model invoking an expansion of the interaction potential based upon rotational invariants [29] has been proposed by Luckhurst and Romano [30]. Their model potential was used later by Longa and Pająk [31] who employed the Ginzburg-Landau phenomenological theory to study the phase behaviour of mesogens with intrinsic biaxiality.

A model for matchbox-symmetric mesogens was used within a density functional approach by Longa et al. [32]. The impressive richness of the phase diagram of matchboxsymmetric hard mesogens was demonstrated by Martínez-Ratón [33]. Their study is based upon fundamental measure theory for the contribution of the hard-repulsive interactions to the excess free energy.

In this work we utilise an off-lattice model for a liquid crystal with intrinsic biaxiality. The molecular architecture assumes matchbox-symmetric mesogens. To obtain an expression for the orientation dependence of the anisotropic interactions we follow Luckhurst and Romano [30] and expand the interaction potential in terms of the rotational invariants proposed by Stone [34].

Based upon symmetry considerations $[21,34]$ and utilising a ladder-operator formalism [29] borrowed from angular-momentum theory in quantum mechanics [35], we can derive an expression for the anisotropic interaction potential in terms of a full contraction of Cartesian second-rank tensors. Thus, our potential is equivalent to the one used in the mean-field calculations of Straley [24] and of Sonnet et al. [5] (see also Ref. 36). We focus on uniaxial and biaxial order parameters [22, 23], the orientation distribution function (odf), and the orientation correlation function.

The odf reflects the symmetry of the mesogens in our model. In addition, we show that at large separations between the mesogens the orientation correlations can be expressed in terms of the uniaxial and biaxial order parameters with a subtle coupling between the two. Such a coupling obviously does not occur in uniaxial nematic phases where much simpler expressions are obtained [37]. Neither the odf nor the orientation correlation function have been considered in earlier work for similar systems. Both quantities turn out to provide a lot of insight into phase changes in this model.

The remainder of the manuscript is organised as follows. Our model is introduced in Section 2. Important mathematical details of the derivation of the anisotropic contribution to the interaction potential are deferred to Appendix A. We give details of the mathematical manipulations here because they are difficult to extract from the existing literature. In Section 3 we introduce key quantities that we intend to analyse in the subsequent Section 4. The paper concludes in Section 5 where we put our results into perspective. Details of the analysis of the asymptotic decay of the orientation correlation functions are given in Appendix B.

\section{Model}

We consider a liquid crystal composed of $N$ mesogens. The interaction between mesogens 1 and 2, whose centres of mass are located at $\boldsymbol{r}_{1}$ and $\boldsymbol{r}_{2}$, is assumed to be pairwise additive. We decompose the interaction potential $u$ into an isotropic part $u_{\text {iso }}$ and into an anisotropic contribution $u_{\text {aniso }}$ according to

$$
u\left(\boldsymbol{r}_{12}, \Omega_{1}, \Omega_{2}\right)=u_{\text {iso }}\left(r_{12}\right)+u_{\text {aniso }}\left(\boldsymbol{r}_{12}, \Omega_{1}, \Omega_{2}\right),
$$


where $r_{12}=\left|\boldsymbol{r}_{12}\right|=\left|\boldsymbol{r}_{1}-\boldsymbol{r}_{2}\right|$ is the distance between the centres of mass. In Equation (1), $\Omega_{1,2}$ are Euler angles. We adopt the convention of Gray and Gubbins [38] for the order in which the rotations about specific axes are being carried out (see Section A.2 of Ref 38). Therefore, $\Omega=(\phi \theta \chi)$ where $\phi$ and $\theta$ refer to the azimuthal and polar angle, respectively.

For $u_{\text {iso }}$ we adopt the soft-sphere potential which permits us to express $u_{\text {iso }}$ as

$$
u_{\text {iso }}\left(r_{12}\right)=4 \varepsilon\left(\frac{\sigma}{r_{12}}\right)^{12}
$$

where, for the time being, $\varepsilon$ and $\sigma$ are just two parameters that will be given a lucid interpretation shortly. To derive an expression for $u_{\text {aniso }}$ we follow Stone [34] who noted that for molecules of arbitrary shape an expansion of $u_{\text {aniso }}$ in a complete set of rotational invariants according to

$$
\begin{aligned}
u_{\text {aniso }}\left(\boldsymbol{r}_{12}, \Omega_{1}, \Omega_{2}\right)= & \sum_{l_{1} l_{2} l} \sum_{n_{1} n_{2}} u_{l_{1} l_{2} l}^{n_{1} n_{2}}\left(r_{12}\right) \\
& \times \mathcal{S}_{l_{1} l_{2} l}^{n_{1} n_{2}}\left(\Omega_{1} \Omega_{2}, \Omega_{12}\right),
\end{aligned}
$$

is generally feasible. In Equation (3), $\left\{u_{l_{1} l_{2} l}^{n_{1} n_{2}}\right\}$ is a set of expansion coefficients that depend only on $r_{12}$, and $\Omega_{12}=\left(\phi_{12} \theta_{12} 0\right)$ describes the orientation of $\widehat{\boldsymbol{r}}_{12}=\boldsymbol{r}_{12} / r_{12}$ in the space-fixed reference frame where $\widehat{\boldsymbol{r}}_{12}$ is pointing from the centre of mass of mesogen 1 to that of mesogen 2 . Here and below we use the caret to indicate a unit vector. The quantity $l^{\prime}$ (that is $l_{1}, l_{2}$, or $l$ ) is a non-negative integer (zero included) and $m^{\prime}$ and $n^{\prime}$ are related to $l^{\prime}$ through the relation $m^{\prime}, n^{\prime} \in\left[-l^{\prime}, l^{\prime}\right]$.

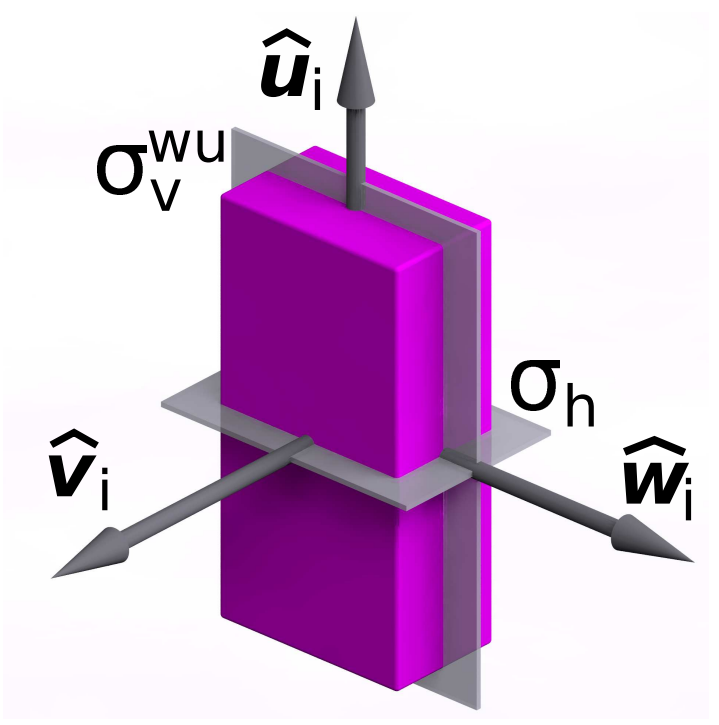

Figure 1. Cartoon of a matchbox-symmetric mesogen for which the major symmetry axis $\widehat{\boldsymbol{u}}_{i}$ and the minor symmetry axis $\widehat{\boldsymbol{v}}_{i}$ are given in Equations (11a) and (11b), respectively; the direction of the second minor symmetry axis is obtained from $\widehat{\boldsymbol{w}}_{i}=\widehat{\boldsymbol{u}}_{i} \times \widehat{\boldsymbol{v}}_{i}$. The mesogen contains reflection planes $\sigma_{\mathrm{h}}$ and $\sigma_{\mathrm{v}}^{w u}$ in the notation of Stone (see Table 2 of Ref. 34). Both reflection planes contain the vector $\widehat{\boldsymbol{w}}_{i}$. A third reflection plane $\sigma_{\mathrm{v}}^{v u}$, orthogonal to $\sigma_{\mathrm{h}}$ and $\sigma_{\mathrm{v}}^{w u}$, is not shown. 


Table 1. Val-
ues of $l, n_{1}$, and
$n_{2}$ to be con-
sidered in Equa-
tion (6).
\begin{tabular}{ccc} 
& \\
\hline$l$ & $n_{1}$ & $n_{2}$ \\
\hline 0 & 0 & 0 \\
2 & 0 & 0 \\
& \pm 2 & 0 \\
0 & \pm 2 \\
& \pm 2 & \pm 2
\end{tabular}

Moreover, in Equation (3) we introduce the scalar function [34]

$$
\begin{aligned}
\mathcal{S}_{l_{1} l_{2} l}^{n_{1} n_{2}}\left(\Omega_{1}, \Omega_{2}, \Omega_{12}\right)= & i^{l_{1}-l_{2}-l} \sum_{m_{1} m_{2} m}\left(\begin{array}{ccc}
l_{1} & l_{2} & l \\
m_{1} & m_{2} & m
\end{array}\right) \\
& \times \mathcal{D}_{m_{1} n_{1}}^{l_{1}}\left(\Omega_{1}\right) \mathcal{D}_{m_{2} n_{2}}^{l_{2}}\left(\Omega_{2}\right) \mathcal{D}_{m 0}^{l^{*}}\left(\Omega_{12}\right),
\end{aligned}
$$

where $i \equiv \sqrt{-1},(\cdots)$ is a Wigner $3 j$ symbol,

$$
\mathcal{D}_{m n}^{l}(\Omega)=e^{-i m \phi} d_{m n}^{l}(\theta) e^{-i n \chi}
$$

is a Wigner rotation matrix, and the asterisk denotes the complex conjugate [35, 38, 39]. The real matrix $d_{m n}^{l}$ depends only on the Euler angle $\theta$; for $l \leq 2$ it is given by Equations (A.114) and (A.115) of the book by Gray and Gubbins [38]. The asymmetric phase factor in front of the summation signs serves to make $\mathcal{S}_{l_{1} l_{2} l}^{n_{1} n_{2}}$ real for $n_{1}=n_{2}=0$ regardless of $l_{1}, l_{2}$, and $l[34]$.

We now specialise the above treatment to matchbox-symmetric particles illustrated by the cartoon presented in Figure 1. Each mesogen possesses three symmetry axes: the major one labelled $\widehat{\boldsymbol{u}}_{i}$ and two minor ones that we refer to as $\widehat{\boldsymbol{v}}_{i}$ and $\widehat{\boldsymbol{w}}_{i}$. These three axes are obviously pairwise orthogonal to one another. Because of the matchbox symmetry, each mesogen contains three reflection planes. Two of these labelled $\sigma_{\mathrm{h}}$ and $\sigma_{\mathrm{v}}^{w u}$ in Figure 1 contain the minor symmetry axis $\widehat{\boldsymbol{w}}_{i}$. According to Figure $1, \widehat{\boldsymbol{u}}_{i}$ is a $\mathcal{C}_{2}$ rotation axis (and so are $\widehat{\boldsymbol{v}}_{i}$ and $\widehat{\boldsymbol{w}}_{i}$ ). Therefore, the matchbox-symmetric mesogens pertain to the point group $\mathrm{D}_{2 \mathrm{~h}}$.

The existence of these symmetry elements has consequences for the expansion coefficients in Equation (3). From Figure 1 one readily sees that each mesogen is centrosymmetric. According to Stone [34], $l$ in Equation (4) must therefore be even. Moreover, the existence of the reflection plane $\sigma_{\mathrm{h}}$ (see Figure 1) then requires $n_{1}$ and $n_{2}$ to be even as well [34].

In addition, we assume $u_{\text {aniso }}$ to depend only on $r_{12}$. This implies that in Equation (4), $l=m=0$. Because of this assumption, the $3 j$ symbol in Equation (42) is nonzero only for $l_{1}=l_{2}$ and $m_{1}=-m_{2}$. This follows from the two selection rules

- $\left|l_{1}-l_{2}\right| \leq l \leq l_{1}+l_{2}$ (triangle inequality) and

- $m_{1}+m_{2}=m$

which need to be satisfied simultaneously. On account of these considerations the triple sum in Equation (4) collapses to a single one; the same is true for the triple sum in Equation (3).

However, the sum on $m$ in Equation (4) involves $2 l+1$ summands and thus becomes 
overwhelmingly large rather quickly as $l$ increases. Hence, we restrict the discussion to $l \leq 2$ from now on and rewrite Equation (3) as

$$
u_{\text {aniso }}\left(r_{12}, \Omega_{1}, \Omega_{2}\right)=\sum_{l=0}^{2} \sum_{n_{1} n_{2}} u_{l l 0}^{n_{1} n_{2}}\left(r_{12}\right) \mathcal{S}_{l l 0}^{n_{1} n_{2}}\left(\Omega_{1}, \Omega_{2}, \Omega_{12}\right)
$$

keeping the argument $\Omega_{12}$ of the $\left\{\mathcal{S}_{l l 0}^{n_{1} n_{2}}\right\}$ only formally. Hence, in principle we need to consider in Equation (6) the terms corresponding to the entries in Table 1. However, the ten terms listed in Table 1 can be reduced by additional symmetry considerations $[21,30]$ as we explain in the following.

We begin with the simplest case $l=n_{1}=n_{2}=0$. Because $\mathcal{S}_{000}^{00}=1$, the leading term in the expansion in Equation (6) is $u_{000}^{00}$. However, we cannot take recourse to any additional first-principles argument to determine what this expansion coefficient should be. We therefore assume that our mesogens interact via dispersion forces. Under this premise, Gray and Gubbins [38] pointed out that $u_{000}^{00} \propto r_{12}^{-6}$. The coefficient of proportionality has dimensions of energy $\times(\text { length })^{-6}$ [see, for example, Equation (2.226) of Ref. 38]. This prompts us to introduce

$$
u_{000}^{00}\left(r_{12}\right)=-4 \varepsilon\left(\frac{\sigma}{r_{12}}\right)^{6} .
$$

Moreover, from Equation (2.234) of Ref. 38 it is clear that all the other expansion coefficients of $u_{\text {aniso }}$ should have the same distance dependence as long as we restrict the discussion to non-polarisable mesogens interacting via dispersion forces only.

Next, we consider $l=2$ but $n_{1}=n_{2}=0$. As we shall demonstrate in Appendix A this contribution refers to a coupling between purely uniaxial degrees of freedom which we shall keep. The next two cases, namely $l=2, n_{1}= \pm 2$ and $n_{2}=0$ as well as $n_{1}=0$ and $n_{2}= \pm 2$ are disregarded because they would entail a coupling between uniaxial and biaxial degrees of freedom as we shall demonstrate also in Appendix A. We deliberately omit such coupling in the present work.

Finally, we include the cases $l=2, n_{1}= \pm 2, n_{2}= \pm 2$. The notation indicates that all four combinations of negative and positive values of $n_{1}$ and $n_{2}$ have to be considered. The corresponding terms in our expression for $u_{\text {aniso }}$ can be simplified by additional symmetry considerations. For example, according to Table 2 in the paper by Stone [29],

$$
\begin{aligned}
& u_{220}^{\frac{22}{2}\left(r_{12}\right)}=u_{220}^{22}\left(r_{12}\right) \\
& u \frac{22}{220}\left(r_{12}\right)=u_{220}^{2 \frac{2}{2}}\left(r_{12}\right),
\end{aligned}
$$

where we used the shorthand notation $\underline{x}=-x$. The separate equalities given in Equations (8a) and (8b) follow from the existence of the reflection plane $\sigma_{\mathrm{v}}^{w u}$ (see Figure 1) $[21,30]$. Reminding ourselves that the mesogens are identical, the expansion coefficients in Equation (6) satisfy one last equality namely $u_{220}^{22}=u_{220}^{22}$ from which it follows that all four expansion coefficients in the Equations (8) are equal. Because of these additional symmetry considerations the number of entries listed in Table 1 is finally reduced to only 5 . Thus, for the case $n_{1}= \pm 2, n_{2}= \pm 2$ the expansion coefficients in Equation (6) are all the same. This allows us to combine the associated rotational invariants 
and introduce

$$
\mathcal{S}_{220}^{ \pm 2 \pm 2}=\mathcal{S}_{220}^{22}+\mathcal{S}_{220}^{22}+\mathcal{S}_{220}^{22}+\mathcal{S}_{220}^{22}
$$

Rather than expressing $u_{\text {aniso }}$ in terms of rotational invariants $\left\{\mathcal{S}_{220}^{n_{1} n_{2}}\right\}$ it will turn out to be more useful to express the anisotropic interaction potential in terms of suitably defined Cartesian tensors. Let us now introduce the traceless ${ }^{1}$, second-rank tensors

$$
\begin{aligned}
& \mathbf{U}\left(\Omega_{i}\right) \equiv \mathbf{U}_{i}=\widehat{\boldsymbol{u}}\left(\Omega_{i}\right) \widehat{\boldsymbol{u}}\left(\Omega_{i}\right)-\frac{1}{3} \mathbf{1} \\
& \mathbf{B}\left(\Omega_{i}\right) \equiv \mathbf{B}_{i}=\widehat{\boldsymbol{v}}\left(\Omega_{i}\right) \widehat{\boldsymbol{v}}\left(\Omega_{i}\right)-\widehat{\boldsymbol{w}}\left(\Omega_{i}\right) \widehat{\boldsymbol{w}}\left(\Omega_{i}\right), \quad i=1,2,
\end{aligned}
$$

where $\mathbf{1}$ is the unit tensor and

$$
\begin{aligned}
& \widehat{\boldsymbol{u}}\left(\Omega_{i}\right) \equiv \widehat{\boldsymbol{u}}_{i}=\left(\begin{array}{c}
\sin \theta_{i} \cos \phi_{i} \\
\sin \theta_{i} \sin \phi_{i} \\
\cos \theta_{i}
\end{array}\right) \\
& \widehat{\boldsymbol{v}}\left(\Omega_{i}\right) \equiv \widehat{\boldsymbol{v}}_{i}=\left(\begin{array}{c}
\cos \phi_{i} \cos \theta_{i} \cos \chi_{i}-\sin \phi_{i} \sin \chi_{i} \\
\sin \phi_{i} \cos \theta_{i} \cos \chi_{i}+\cos \phi_{i} \sin \chi_{i} \\
-\sin \theta_{i} \cos \chi_{i}
\end{array}\right)
\end{aligned}
$$

represent the orientation of the molecular major and minor symmetry axes in a spacefixed coordinate system. The tensors $\mathbf{U}$ and $\mathbf{B}$ are referred to as the uniaxial and biaxial tensors, respectively. The terms involving products of $\widehat{\boldsymbol{u}}, \widehat{\boldsymbol{v}}$, and $\widehat{\boldsymbol{w}}$ on the right-hand sides of Equations (10a) and (10b) are to by understood as dyads.

The third vector is defined through the expression $\widehat{\boldsymbol{w}}_{i}=\widehat{\boldsymbol{u}}_{i} \times \widehat{\boldsymbol{v}}_{i}$. Using the expressions in Equations (2) and (6)-(10) we can eventually cast $u$ in Equation (1) as

$$
u\left(r_{12}, \Omega_{1}, \Omega_{2}\right)=u_{\mathrm{LJ}}\left(r_{12}\right)-4 \varepsilon\left(\frac{\sigma}{r_{12}}\right)^{6} \Psi\left(\Omega_{12}\right),
$$

where

$$
u_{\mathrm{LJ}}=4 \varepsilon\left[\left(\frac{\sigma}{r_{12}}\right)^{12}-\left(\frac{\sigma}{r_{12}}\right)^{6}\right]
$$

is the well-known Lennard-Jones potential,

$$
\Psi\left(\Omega_{12}\right)=\varepsilon^{\prime} \mathbf{U}_{1}: \mathbf{U}_{2}+\varepsilon^{\prime \prime} \mathbf{B}_{1}: \mathbf{B}_{2}
$$

is the anisotropy function, and $\mathbf{X}: \mathbf{X}=X_{\alpha \beta} X_{\beta \alpha}(\mathbf{X}=\mathbf{U}$ or $\mathbf{B})$ denotes the full contraction of two second-rank tensors using the Einstein repeated summation-index convention.

At this point we emphasise that the expressions given in Equations (12)-(14) do by no means allow for modeling mesogens that have the shape of matchboxes. Rather the interaction potential possesses certain symmetry elements such as $n$-fold rotation axes and reflection planes that would be characteristic of a real matchbox. Therefore, we refer to our mesogens as "matchbox-symmetric".

\footnotetext{
${ }^{1}$ For particularly important properties of traceless matrices, see Ref. [40].
} 
In Appendix A we link the rotational invariants introduced in Equation (4) to the Cartesian tensors $\mathbf{U}$ and $\mathbf{B}$ in Equations (10a) and (10b), respectively. This is accomplished with the ladder-operator technique proposed by Stone [34]. It is identical to the one arising in angular-momentum theory in quantum mechanics [35].

The philosophy of just equipping the interaction potential with certain symmetry elements is not at all new. In fact, it has been employed in the original work by Maier and Saupe [41] in which the orientation dependence of the interaction between uniaxial mesogens is accounted for by the second Legendre polynomial. As pointed out by Schoen et al. [42], for any fixed relative orientation of a pair of mesogens, Maier and Saupe's interaction potential will be isotropic. Nonetheless, properties of uniaxial nematic phases have been successfully reproduced by this minimalistic approach.

Finally, we are in a position to attach physical meaning to the parameters $\varepsilon$ and $\sigma$ introduced in Equation (2) through the relations $u_{\mathrm{LJ}}(\sigma)=0$ and $\mathrm{d} u_{\mathrm{LJ}} /\left.\mathrm{d} r_{12}\right|_{r_{12}=\sigma}=$ 0 . Thus, $\sigma$ is a measure of the diameter of the spherically symmetric core of our mesogens and $\varepsilon$ is the depth of the attractive well. In Equation (14), $\varepsilon^{\prime}$ and $\varepsilon^{\prime \prime}$ are dimensionless coupling constants referring to the strength of the coupling of uniaxial and biaxial degrees of freedom, respectively. In these parameters we lump together all trivial numerical pre-factors arising from the expansion of $u_{\text {aniso }}$ in terms of the rotational invariants $\left\{\mathcal{S}_{l_{1} l_{2} l}^{n_{1} n_{2}}\right\}$ and other molecular constants such as polarizabilities [38].

Throughout this work we fix $\varepsilon^{\prime}=0.375$. This value was chosen according to the following criteria. On the one hand, $\varepsilon^{\prime}$ must not be too large to avoid glassy states before biaxial nematic phases can even form. On the other hand, $\varepsilon^{\prime}$ must not be too small so that stable uniaxial and biaxial nematic phases occur for moderate values of $T$ and $\rho$. In practice, we performed short test runs to fine-tune this parameter according to both criteria.

\section{Orientational structure and order parameters}

\subsection{Orientation-dependent correlation functions}

\subsubsection{Expansion coefficients}

To gain deeper insight into the nature of uniaxial and biaxial nematic order we introduce the orientation-dependent pair correlation function. As our point of departure we choose the two-particle generic distribution function [38] defined as

$$
\rho\left(\boldsymbol{r}_{1}, \boldsymbol{r}_{2}, \Omega_{1}, \Omega_{2}\right)=\rho\left(\boldsymbol{r}_{1}, \Omega_{1}\right) \rho\left(\boldsymbol{r}_{2}, \Omega_{2}\right) g\left(\boldsymbol{r}_{1}, \boldsymbol{r}_{2}, \Omega_{1}, \Omega_{2}\right),
$$

where $g$ is the orientation-dependent pair correlation function and $\rho$ on the right handside denote the generic single-particle distribution functions. Assuming our system to be homogeneous we can rewrite the latter function as

$$
\rho(\boldsymbol{r}, \Omega)=\rho \alpha(\Omega),
$$

where $\rho$ on the right-hand side is the (mean) number density and $\alpha$ is the odf. The odf is normalised according to $\int \mathrm{d} \Omega \alpha=1$. Because homogeneity implies translational 
invariance we can rewrite Equation (15) as

$$
\begin{aligned}
\frac{1}{\rho^{2}} \rho\left(\boldsymbol{r}_{12}, \Omega_{1}, \Omega_{2}\right) & =\alpha\left(\Omega_{1}\right) \alpha\left(\Omega_{2}\right) g\left(\boldsymbol{r}_{12}, \Omega_{1}, \Omega_{2}\right) \\
& =g^{\prime}\left(\boldsymbol{r}_{12}, \Omega_{1}, \Omega_{2}\right),
\end{aligned}
$$

where $g^{\prime}$ is a "reduced" distribution function [37]. It is introduced because in the absence of positional and orientational correlations $g \stackrel{r_{12} \rightarrow \infty}{\longrightarrow} 1$ and therefore $g^{\prime}$ can simply be cast as the product of the two odf's [37].

Similar to Equation (3) we can expand $g^{\prime}$ in terms of rotational invariants via

$$
g^{\prime}\left(\boldsymbol{r}_{12}, \Omega_{1}, \Omega_{2}\right)=\sum_{l_{1} l_{2} l} \sum_{n_{1} n_{2}} g_{l_{1} l_{2} l}^{n_{1} n_{2}}\left(r_{12}\right) \mathcal{S}_{l_{1} l_{2} l}^{n_{1} n_{2}}\left(\Omega_{1}, \Omega_{2}, \Omega_{12}\right),
$$

where $\left\{g_{l_{1} l_{2} l}^{n_{1} n_{2}}\right\}$ is a set of expansion coefficients. We immediately restrict the discussion to the case in which $g$ depends only on $r_{12}$. This implies that $l=m=0$. Employing the two selection rules for nonzero $3 j$ symbols introduced in Section 2 we can replace the $3 j$ symbol by [see Equation (4)]

$$
\left(\begin{array}{ccc}
l & l & 0 \\
m & \underline{m} & 0
\end{array}\right)=\frac{(-)^{l+m}}{\sqrt{2 l+1}}
$$

Using Equation (4) we can rewrite Equation (18) more explicitly as

$$
\begin{aligned}
g^{\prime}\left(r_{12}, \Omega_{1}, \Omega_{2}\right)= & \sum_{l} \frac{1}{\sqrt{2 l+1}}\left(\frac{2 l+1}{8 \pi^{2}}\right)^{2} \sum_{n_{1} n_{2}} g_{l l 0}^{n_{1} n_{2}}\left(r_{12}\right) \\
& \times \sum_{m}(-)^{m} \mathcal{D}_{m n_{1}}^{l *}\left(\Omega_{1}\right) \mathcal{D}_{\underline{m} n_{2}}^{l *}\left(\Omega_{2}\right),
\end{aligned}
$$

where $(-)^{m}=(-1)^{m}$.

The extra factor of $\left(\frac{2 l+1}{8 \pi^{2}}\right)^{2}$ in Equation (20) has been introduced to make sure that the final expression for $g_{l l 0}^{n_{1} n_{2}}$ is compatible with the Cartesian tensor formulation developed in Appendix A. The latter does not take recourse to the orthogonality of the Wigner rotation matrices and thus lacks factors of this sort.

Multiplying both sides of Equation (20) by

$$
\sum_{m}(-)^{m} \mathcal{D}_{m n_{1}}^{l}\left(\Omega_{1}\right) \mathcal{D}_{\underline{m} n_{2}}^{l}\left(\Omega_{2}\right),
$$

integrating over $\mathrm{d} \Omega_{1} \mathrm{~d} \Omega_{2}$, and employing the orthogonality of the Wigner rotation matrices

$$
\int \mathrm{d} \Omega \mathcal{D}_{m n}^{l_{m n}^{*}}(\Omega) \mathcal{D}_{m^{\prime} n^{\prime}}^{l^{\prime}}(\Omega)=\frac{8 \pi^{2}}{2 l^{\prime}+1} \delta_{l^{\prime} l} \delta_{m^{\prime} m} \delta_{n^{\prime} n}
$$

where the $\delta_{i j}$ 's are Kronecker symbols. 
After rearranging terms we then obtain the relation

$$
\begin{aligned}
\sqrt{2 l+1} g_{l l 0}^{n_{1} n_{2}}\left(r_{12}\right)= & \iint \mathrm{d} \Omega_{1} \mathrm{~d} \Omega_{2} g^{\prime}\left(r_{12}, \Omega_{1}, \Omega_{2}\right) \\
& \times \sum_{m}(-)^{m} \mathcal{D}_{m n_{1}}^{l}\left(\Omega_{1}\right) \mathcal{D}_{\underline{m} n_{2}}^{l}\left(\Omega_{2}\right) .
\end{aligned}
$$

Using Equation (4) it is easy to verify that our final expression for the expansion coefficients $g_{l l 0}^{n_{1} n_{2}}$ can be cast as

$$
g_{l l 0}^{n_{1} n_{2}}\left(r_{12}\right)=\iint \mathrm{d} \Omega_{1} \mathrm{~d} \Omega_{2} g^{\prime}\left(r_{12}, \Omega_{1}, \Omega_{2}\right) \mathcal{S}_{l l 0}^{n_{1} n_{2} *} .
$$

\subsubsection{Computer simulations}

Following Zannoni [37, 43], we can rewrite Equation (23) to make it suitable for an evaluation in computer simulations. To that end we divide and multiply by

$$
g_{0}^{\prime}\left(r_{12}\right)=\iint \mathrm{d} \Omega_{1} \mathrm{~d} \Omega_{2} g^{\prime}\left(r_{12}, \Omega_{1}, \Omega_{2}\right)
$$

which permits us to recast Equation $(23)$ as $[42,44]$

$$
g_{l l 0}^{n_{1} n_{2}}\left(r_{12}\right)=g_{0}^{\prime}\left(r_{12}\right)\left\langle\mathcal{S}_{l l 0}^{n_{1} n_{2} *}\right\rangle_{\text {shell }}
$$

where $\langle\ldots\rangle_{\text {shell }} \equiv g_{0}^{\prime-1} \iint \mathrm{d} \Omega_{1} \mathrm{~d} \Omega_{2} g^{\prime} \ldots$ and we dropped the arguments of $\mathcal{S}_{l l 0}^{n_{1} n_{2}}$ to simplify the notation.

Notice that on account of the definition of $g^{\prime}$ in Equation (17), $g_{0}^{\prime}$ is not the normal radial distribution function. For one, $g^{\prime}$ still involves the product of the two odf's and second, the expression in Equation (24) is not defined as an unweighted average over orientations of $g$ introduced in Equation (15). Therefore, $g_{0}^{\prime}$ is unknown at this stage because the odf's are undetermined. However, in practice this is not a problem because orientation correlations are fully accounted for by $\left\langle\mathcal{S}_{l l 0}^{n_{1} n_{2}}\right\rangle_{\text {shell }}$. Thus, if the focus is exclusively on orientational correlations we can consider $\left\langle\mathcal{S}_{l l 0}^{n_{1} n_{2}}\right\rangle_{\text {shell }}$ rather than the expansion coefficient $g_{l l 0}^{n_{1} n_{2}}$ associated with it.

We can now invoke the same symmetry considerations already applied to $\left\{u_{l l 0}^{n_{1} n_{2}}\right\}$ in Section 2. Leaving out the case $l=n_{1}=n_{2}=0$ for obvious reasons, only $l=2$ and $n_{1}=n_{2}=0$ and $n_{1}= \pm 2$ and $n_{2}= \pm 2$ will be considered (see Table 1); the other two cases, namely $n_{1}= \pm 2, n_{2}=0$ as well as $n_{1}=0$ and $n_{2}= \pm 2$ are disregarded because $\Psi$ in Equation (14) does not contain a term corresponding to coupling between uniaxial and biaxial degrees of freedom [see also Equations (A13) and (A14)]. Therefore, we only consider [see Appendix A]

$$
\begin{aligned}
\sqrt{5}\left\langle\mathcal{S}_{220}^{00}\left(\Omega_{1}, \Omega_{2}, \Omega_{12}\right)\right\rangle_{\text {shell }} & =\frac{3}{2}\left\langle\mathbf{U}_{1}: \mathbf{U}_{2}\right\rangle_{\text {shell }} \\
\sqrt{5}\left\langle\mathcal{S}_{220}^{ \pm 2 \pm 2}\left(\Omega_{1}, \Omega_{2}, \Omega_{12}\right)\right\rangle_{\text {shell }} & =\left\langle\mathbf{B}_{1}: \mathbf{B}_{2}\right\rangle_{\text {shell }},
\end{aligned}
$$

where the tensors $\mathbf{U}$ and $\mathbf{B}$ have been defined in Equations (10a) and (10b), respectively. 
A couple of comments apply at this point. For $n_{1}=n_{2}=0, \mathcal{S}_{l l 0}^{00}$ is real and therefore $\mathcal{S}_{l l 0}^{00 *}=\mathcal{S}_{l l 0}^{00}$ so that Equation (26a) follows from the general expression given in Equation (23) without further ado. Using the symmetry property of the Wigner rotation matrices

$$
\mathcal{D}_{\underline{m n}}^{l}(\Omega)=(-)^{m+n} \mathcal{D}_{m n}^{l^{*}}(\Omega)
$$

it follows that

$$
\begin{aligned}
& \sum_{m}(-)^{m} \mathcal{D}_{m n_{1}}^{l}\left(\Omega_{1}\right) \mathcal{D}_{\underline{m} \underline{n}_{2}}^{l}\left(\Omega_{2}\right) \\
= & \sum_{m}(-)^{m} \mathcal{D}_{\underline{m n}_{1}}^{l *}\left(\Omega_{1}\right) \mathcal{D}_{m \underline{n}_{2}}^{l *}\left(\Omega_{2}\right) \\
= & \sum_{m}^{m}(-)^{m} \mathcal{D}_{m \underline{n}_{1}}^{l *}\left(\Omega_{1}\right) \mathcal{D}_{\underline{m n}_{2}}^{l *}\left(\Omega_{2}\right),
\end{aligned}
$$

where we omitted a factor $(-)^{2 m+n_{1}+n_{2}}$ because $n_{1}$ and $n_{2}$ are supposed to be even integers. Thus, it is clear that $\mathcal{S}_{l l 0}^{n_{1} n_{2} *}=\mathcal{S}_{l l 0}^{\underline{n}_{1} \underline{n}_{2}}$ and therefore $\mathcal{S}_{220}^{ \pm 2 \pm 2^{*}}=\mathcal{S}_{220}^{ \pm 2 \pm 2}$ using Equation (9).

Utilising Equations (26) has the additional advantage that in a computer simulation it is rather straightforward to compute $\left\langle\mathcal{S}_{220}^{n_{1} n_{2}}\right\rangle_{\text {shell }}$. Notice that the expressions in Equations (26a) and (26b) clearly indicate that only the relative orientations between mesogens 1 and 2 matter. As explained by Zannoni [43] one could therefore compute $\left\langle\mathcal{S}_{220}^{n_{1} n_{2}}\right\rangle_{\text {shell }}$ as a histogram. According to the distance $r_{12}$ a histogram bin is chosen and the tensors $\mathbf{U}$ and $\mathbf{B}$ are computed for both mesogens of the pair. These tensors are then fully contracted and the resulting values are sorted into the bin corresponding to $r_{12}$. The procedure is repeated for each pair of mesogens in the given configuration. Obviously, this procedure has to be repeated for as many configurations as it may take to compute the histogram with an acceptable statistical accuracy. In closing we note that a procedure very similar to the one described has been employed by Streett and Tildesley [44] and later by Schoen et al. [42].

\subsubsection{The limit of large intermolecular separations}

It is instructive to investigate the expressions given in Equations (26a) and (26b) in the limit $r_{12} \rightarrow \infty$. In this limit, we can rewrite Equation (24) as

$$
\lim _{r_{12} \rightarrow \infty} g_{0}^{\prime}\left(r_{12}\right)=\iint \mathrm{d} \Omega_{1} \mathrm{~d} \Omega_{2} \alpha\left(\Omega_{1}\right) \alpha\left(\Omega_{2}\right)=1,
$$

where we also used Equation (17). The previous result follows because the odf's are normalised and because we assumed that $\lim _{r_{12} \rightarrow \infty} g=1$ [37]. This hypothesis is certainly valid in an isotropic phase; in an ordered phase, however, the assumption can only be justified a posteriori. This is because $\lim _{r_{12} \rightarrow \infty} g=1$ neglects all (spatial and orientational) correlations between mesogens 1 and 2 which is difficult to justify at the outset.

However, accepting the above approximation as a working hypothesis for the time 
being we use Equation (23) and introduce

$$
\begin{aligned}
\lim _{r_{12} \rightarrow \infty} g_{220}^{n_{1} n_{2}}\left(r_{12}\right)= & \left\langle\mathcal{S}_{220}^{n_{1} n_{2}}\right\rangle_{\infty} \\
= & \iint \mathrm{d} \Omega_{1} \mathrm{~d} \Omega_{2} \alpha\left(\Omega_{1}\right) \alpha\left(\Omega_{2}\right) \mathcal{S}_{220}^{n_{1} n_{2} *} \\
= & \frac{1}{\sqrt{5}} \iint \mathrm{d} \Omega_{1} \mathrm{~d} \Omega_{2} \alpha\left(\Omega_{1}\right) \alpha\left(\Omega_{2}\right) \\
& \times \sum_{m}(-)^{m} \mathcal{D}_{m n_{1}}^{2}\left(\Omega_{1}\right) \mathcal{D}_{\underline{m} n_{2}}^{2}\left(\Omega_{2}\right) .
\end{aligned}
$$

We dropped the word "shell" as an index because in the limit $r_{12} \rightarrow \infty$ the shell loses its meaning. In fact, $g_{220}^{n_{1} n_{2}}$ will assume a constant value irrespective of how one defines a shell.

We now follow Mulder [21] and expand the odf according to

$$
\begin{aligned}
\alpha(\Omega) & =\sum_{l^{\prime} m^{\prime} n^{\prime}} \alpha_{m^{\prime} n^{\prime}}^{l^{\prime}} \mathcal{Q}_{m^{\prime} n^{\prime}}^{l^{\prime}}(\Omega) \\
& =\sum_{l m n} \alpha_{|m||n|}^{l} s_{|m||n|} \mathcal{D}_{m n}^{l}(\Omega),
\end{aligned}
$$

where $m^{\prime}, n^{\prime} \in[0, l]$; instead, $n$ and $m$ are defined as usual (see Section 2). In addition, the symmetry of the odf requires $l, m$, and $n$ to be even. The functions $\left\{\mathcal{Q}_{m^{\prime} n^{\prime}}^{l}\right\}$ are defined in Equation (3.5) of the paper by Mulder [21]. The coefficient

$$
s_{m n}=\left(\frac{1}{\sqrt{2}}\right)^{2+\delta_{m 0}+\delta_{n 0}} 2^{\delta_{m 0}+\delta_{n 0}}=2^{\left(\delta_{m 0}+\delta_{n 0}-2\right) / 2}
$$

serves to make the expression on the second line of Equation (31) consistent with Mulder's Equations (3.5) and (3.6). The coefficients $\left\{\alpha_{m n}^{l}\right\}$ are expansion coefficients [21-24, 37, 43]. The expansion coefficients should not be confused with order parameters because the latter are defined on the interval $[0,1]$ whereas this is not necessarily true for the former,

We now insert Equations (31) and (32) into Equation (30) and obtain eventually [cf., Equations (26)]

$$
\begin{aligned}
\sqrt{5}\left\langle\mathcal{S}_{220}^{00}\right\rangle_{\infty} & =\frac{3}{2}\left\langle\mathbf{U}_{1}: \mathbf{U}_{2}\right\rangle_{\infty}=\left(\alpha_{00}^{2}\right)^{2}+\left(\alpha_{20}^{2}\right)^{2} \\
\sqrt{5}\left\langle\mathcal{S}_{220}^{ \pm 2 \pm 2}\right\rangle_{\infty} & =\left\langle\mathbf{B}_{1}: \mathbf{B}_{2}\right\rangle_{\infty}=2\left[\left(\alpha_{22}^{2}\right\rangle^{2}+\left\langle\alpha_{02}^{2}\right)^{2}\right]
\end{aligned}
$$

where we dropped all arguments to simplify the notation. Details of this derivation have been deferred to Appendix B. Our analysis also enables us to evaluate $\left\langle\mathcal{S}_{220}^{ \pm 20}\right\rangle_{\infty}$ and $\left\langle\mathcal{S}_{220}^{0 \pm 2}\right\rangle_{\infty}$ where a definition analogous to the one for $\mathcal{S}_{220}^{ \pm 2 \pm 2}$ in Equation (9) is used. Based upon Equations (A13) and (A14) we find that

$$
\begin{aligned}
\sqrt{5}\left\langle\mathcal{S}_{220}^{ \pm 20}\right\rangle_{\infty} & =\sqrt{\frac{3}{2}}\left\langle\mathbf{U}_{1}: \mathbf{B}_{2}\right\rangle_{\infty}=\sqrt{\frac{3}{2}}\left\langle\mathbf{B}_{1}: \mathbf{U}_{2}\right\rangle_{\infty} \\
& =\sqrt{2}\left[\alpha_{00}^{2} \alpha_{02}^{2}+\alpha_{22}^{2} \alpha_{20}^{2}\right] .
\end{aligned}
$$


Together the expressions in Equations (33) and (34) reflect the coupling between uniaxial and biaxial degrees of freedom. However, from the definition of $\Psi$ in Equation (14) it is clear that we deliberately disregard such coupling. According to one's physical intuition one would therefore expect $\mathbf{B}_{1}: \mathbf{U}_{2}=\mathbf{U}_{1}: \mathbf{B}_{2}=0$ even in uniaxial or biaxial nematic phases. If this is indeed so $\alpha_{20}^{2}=\alpha_{02}^{2}=0$ and therefore we obtain from Equations (33) our final result

$$
\begin{aligned}
\sqrt{5}\left\langle\mathcal{S}_{220}^{00}\right\rangle_{\infty} & =S^{2} \\
\sqrt{5}\left\langle\mathcal{S}_{220}^{ \pm 2 \pm 2}\right\rangle_{\infty} & =2 \eta^{2}
\end{aligned}
$$

where the nematic and biaxial order parameters $S$ and $\eta$ will be defined below in Equations (42) and (50). Equations (35) are derived by comparing Equation (3.9) in Mulder's paper with our Equations (42) and (50) and by noticing that our expansion coefficients $\left\{\alpha_{m n}^{l}\right\}$ have to be identical to Mulder's expansion coefficients $\left\{q_{m n}^{l}\right\}$ [21]. We defer a study of the effects of including the coupling between uniaxial and biaxial degrees of freedom to a separate publication.

\subsection{Rotations of the eigensystems}

Another quantity of interest to us is the odf. In computer simulations the odf can be computed as a two-dimensional histogram of the angles $\theta_{i}$ and $\phi_{i}$ describing the orientation $\widehat{\boldsymbol{u}}_{i}$ of mesogen $i$ [see Equation (11a)]. Hence, the tips of the vectors $\left\{\widehat{\boldsymbol{u}}_{i}\right\}$ correspond to points on $\mathcal{S}^{2}$ (i.e., the surface of the unit sphere). To compute the odf, $\mathcal{S}^{2}$ is partitioned into small bins of size $\delta A=\delta \phi \times \delta \theta$. One then simply counts the number of mesogens whose orientation pertains to a specific bin. Clearly, the size of the bins is largest along the equator of $\mathcal{S}^{2}$ and becomes progressively smaller towards its poles if the number of bins stays fixed.

Suppose now the liquid crystal is in a uniaxial nematic phase. Then the vectors $\left\{\widehat{\boldsymbol{u}}_{i}\right\}$ are predominantly aligned with $\widehat{\boldsymbol{n}}$ where the tip of $\widehat{\boldsymbol{n}}$ can be represented by a point on $\mathcal{S}^{2}$ as well. In the absence of external fields, this point can lie anywhere on $\mathcal{S}^{2}$. In particular, it can be located near the poles. This implies that most of the $\left\{\widehat{\boldsymbol{u}}_{i}\right\}$ also correspond to points in that region of $\mathcal{S}^{2}$. Thus, if the histogram is computed, the odf would only be accessible with rather low resolution, or, if the resolution is enhanced by making $\delta A$ smaller, with insufficient statistical accuracy.

It is therefore advantageous to rotate the vectors $\left\{\widehat{\boldsymbol{u}}_{i}\right\}$ (or, alternatively, the basis used to define $\mathcal{S}^{2}$ ) such that the orientations of the mesogens are sorted into bins located at or near the equator of $\mathcal{S}^{2}$. We can determine a basis optimised according to the above criteria following the protocol originally proposed by Camp and Allen [23] to define suitable order parameters.

Let us introduce three instantaneous, second-rank, symmetric, and traceless tensors

$$
\mathbf{Q}_{x x}=\frac{1}{2 N} \sum_{i=1}^{N}\left(3 \widehat{\boldsymbol{x}}_{i} \widehat{\boldsymbol{x}}_{i}-\mathbf{1}\right),
$$

where $\widehat{\boldsymbol{x}}_{i}$ represents $\widehat{\boldsymbol{u}}_{i}, \widehat{\boldsymbol{v}}_{i}$, or $\widehat{\boldsymbol{w}}_{i}$. The three tensors $\mathbf{Q}_{x x}$ satisfy the eigenvalue equation

$$
\mathbf{Q}_{x x} \widehat{\boldsymbol{q}}_{ \pm, 0}^{x}=\lambda_{ \pm, 0}^{x} \widehat{\boldsymbol{q}}_{ \pm, 0}^{x},
$$

where $\lambda_{-}^{x} \leq \lambda_{0}^{x}<\lambda_{+}^{x}$ are the three eigenvalues and $\widehat{\boldsymbol{q}}_{ \pm, 0}^{x}$ are the associated eigenvectors 
where the equal sign applies if the nematic phase is uniaxial. Equation (37) gives us a total of nine eigenvalues and associated -vectors. It has been pointed out by Mulder [21] that on account of symmetry arguments the three tensors $\mathbf{Q}_{u u}, \mathbf{Q}_{v v}$ and $\mathbf{Q}_{w w}$ share their eigensystem and can thus be diagonalised simultaneously. However, the role each of the three eigenvectors is playing depends on the symmetry axis one is referring to. This is illustrated by the sketch in Figure 2 where the polar angle $\theta_{v}$ is formed between $\widehat{\boldsymbol{v}}_{i}$ and the axis $\widehat{\boldsymbol{b}}_{3}$; the azimuthal angle $\varphi_{v}$ is formed between the projection of $\widehat{\boldsymbol{v}}_{i}$ onto the $\widehat{\boldsymbol{b}}_{1}-\widehat{\boldsymbol{b}}_{2}$ plane and the $\widehat{\boldsymbol{b}}_{1}$-axis.

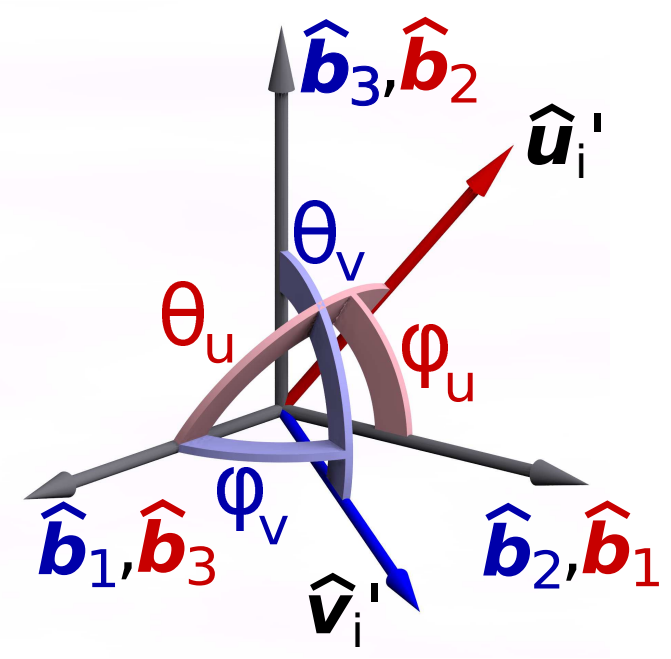

Figure 2. Sketch of the eigensystem $\left(\widehat{\boldsymbol{b}}_{1}, \widehat{\boldsymbol{b}}_{2}, \widehat{\boldsymbol{b}}_{3}\right)$ (see text). Blue symbols are defined with respect to $\widehat{\boldsymbol{v}}_{i}^{\prime}$ whereas symbols in red refer to $\widehat{\boldsymbol{u}}_{i}^{\prime}$. Polar and azimuthal angles $\theta_{v}$ and $\varphi_{v}$ of the symmetry axis $\widehat{\boldsymbol{v}}_{i}^{\prime}$ are defined as indicated in the figure. The role played by the three eigenvectors changes if the symmetry axis $\widehat{\boldsymbol{u}}_{i}^{\prime}$ is considered as indicated such that $\widehat{\boldsymbol{b}}_{1} \rightarrow \widehat{\boldsymbol{b}}_{3}, \widehat{\boldsymbol{b}}_{2} \rightarrow \widehat{\boldsymbol{b}}_{1}$, and $\widehat{\boldsymbol{b}}_{3} \rightarrow \widehat{\boldsymbol{b}}_{2}$ (red symbols) and the corresponding angles $\theta_{u}$ and $\varphi_{u}$ are defined accordingly. The transformation $\left(\widehat{\boldsymbol{b}}_{1}, \widehat{\boldsymbol{b}}_{2}, \widehat{\boldsymbol{b}}_{3}\right) \rightarrow\left(\widehat{\boldsymbol{b}}_{2}, \widehat{\boldsymbol{b}}_{3}, \widehat{\boldsymbol{b}}_{1}\right)$ preserves the right-handedness of the eigensystem.

We define as $\widehat{\boldsymbol{b}}_{3}$ the eigenvector corresponding to the eigenvalue $\lambda_{+}^{u}$. Similarly, we take $\widehat{\boldsymbol{b}}_{1}$ to be the eigenvector corresponding to the eigenvalue $\lambda_{+}^{v}$. Last but not least, $\widehat{\boldsymbol{b}}_{2}=\widehat{\boldsymbol{b}}_{3} \times \widehat{\boldsymbol{b}}_{1}$. Let us now introduce the standard basis $\left(\widehat{\boldsymbol{e}}_{\mathrm{x}}, \widehat{\boldsymbol{e}}_{\mathrm{y}}, \widehat{\boldsymbol{e}}_{\mathrm{z}}\right)$, where $\widehat{\boldsymbol{e}}_{\mathrm{x}}^{\mathrm{T}}=(1,0,0)$, $\widehat{\boldsymbol{e}}_{\mathrm{y}}^{\mathrm{T}}=(0,1,0), \widehat{\boldsymbol{e}}_{\mathrm{z}}^{\mathrm{T}}=(0,0,1)$ and $\mathrm{T}$ denotes the transpose. We can then transform the vectors $\widehat{\boldsymbol{u}}_{i}$ and $\widehat{\boldsymbol{v}}_{i}$ given in the standard basis to new vectors $\widehat{\boldsymbol{u}}_{i}^{\prime}$ and $\widehat{\boldsymbol{v}}_{i}^{\prime}$ using the three eigenvectors $\widehat{\boldsymbol{b}}_{1}, \widehat{\boldsymbol{b}}_{2}$, and $\widehat{\boldsymbol{b}}_{3}$ as the new reference frame. The transformations can be cast as

$$
\begin{aligned}
& \widehat{\boldsymbol{u}}_{i}^{\prime}=\mathbf{R}_{u u} \widehat{\boldsymbol{u}}_{i} \\
& \widehat{\boldsymbol{v}}_{i}^{\prime}=\mathbf{R}_{v v} \widehat{\boldsymbol{v}}_{i}, \quad i=1, \ldots, N,
\end{aligned}
$$

where $\widehat{\boldsymbol{u}}_{i}^{\prime}$ and $\widehat{\boldsymbol{v}}_{i}^{\prime}$ depend on $\varphi_{u, v}$ and $\theta_{u, v}$ as illustrated by the sketch in Figure 2. Note, that $\varphi_{u, v}$ and $\theta_{u, v}$ themselves are functions of the three Euler angles $\phi_{i}, \theta_{i}$, and $\chi_{i}$. In Equations (38), $\mathbf{R}_{u u}$ and $\mathbf{R}_{v v}$ are respective direction-cosine matrices between the vectors of the standard basis and $\left(\widehat{\boldsymbol{b}}_{2}, \widehat{\boldsymbol{b}}_{3}, \widehat{\boldsymbol{b}}_{1}\right)$ (reference to $\widehat{\boldsymbol{u}}_{i}$ ) on the one hand and $\left(\widehat{\boldsymbol{b}}_{1}, \widehat{\boldsymbol{b}}_{2}, \widehat{\boldsymbol{b}}_{3}\right.$ ) (reference to $\widehat{\boldsymbol{v}}_{i}$ ) on the other hand. 
The relevant angles can then be computed via (see Figure 2)

$$
\begin{aligned}
\cos \theta_{u, i} & =\widehat{\boldsymbol{u}}_{i}^{\prime} \cdot \widehat{\boldsymbol{b}}_{1} \\
\cos \theta_{v, i} & =\widehat{\boldsymbol{v}}_{i}^{\prime} \cdot \widehat{\boldsymbol{b}}_{3}
\end{aligned}
$$

and

$$
\begin{aligned}
& \cos \varphi_{u, i}= \frac{\left(\widehat{\boldsymbol{b}}_{2} \widehat{\boldsymbol{b}}_{2}+\widehat{\boldsymbol{b}}_{3} \widehat{\boldsymbol{b}}_{3}\right) \widehat{\boldsymbol{u}}_{i}^{\prime} \cdot \widehat{\boldsymbol{b}}_{1}}{\left|\left(\widehat{\boldsymbol{b}}_{2} \widehat{\boldsymbol{b}}_{2}+\widehat{\boldsymbol{b}}_{3} \widehat{\boldsymbol{b}}_{3}\right) \widehat{\boldsymbol{u}}_{i}^{\prime}\right|} \\
& \cos \varphi_{v, i}=\frac{\left(\widehat{\boldsymbol{b}}_{1} \widehat{\boldsymbol{b}}_{1}+\widehat{\boldsymbol{b}}_{2} \widehat{\boldsymbol{b}}_{2}\right) \widehat{\boldsymbol{v}}_{i}^{\prime} \cdot \widehat{\boldsymbol{b}}_{2}}{\left|\left(\widehat{\boldsymbol{b}}_{1} \widehat{\boldsymbol{b}}_{1}+\widehat{\boldsymbol{b}}_{2} \widehat{\boldsymbol{b}}_{2}\right) \widehat{\boldsymbol{v}}_{i}^{\prime}\right|} .
\end{aligned}
$$

The tensors in parentheses in Equations (40a) and (40b) effect a projection of $\widehat{\boldsymbol{u}}_{i}^{\prime}$ and $\widehat{\boldsymbol{v}}_{i}^{\prime}$ onto the $\widehat{\boldsymbol{b}}_{2}-\widehat{\boldsymbol{b}}_{3}$ and $\widehat{\boldsymbol{b}}_{1}-\widehat{\boldsymbol{b}}_{2}$ planes, respectively.

With the aid of Equations (39a) and (40a) on the one hand and (39b) and (40b) on the other hand we are in a position to compute the odf from the expression

$$
\alpha_{u, v}(\omega)=\frac{\sum_{i=1}^{N}\left\langle\delta\left(\omega-\omega_{i}^{\prime}\right)\right\rangle}{\int_{0}^{2 \pi} \int_{0}^{\pi} \sin \theta \mathrm{d} \theta \mathrm{d} \varphi \sum_{i=1}^{N}\left\langle\delta\left(\omega-\omega_{i}^{\prime}\right)\right\rangle},
$$

where $\langle\ldots\rangle$ denotes an ensemble average [7], $\delta$ is the Dirac $\delta$-function, and $\delta\left(\omega-\omega_{i}^{\prime}\right)$ is shorthand notation for $\delta\left(\theta-\theta_{i}^{\prime}\right) \delta\left(\varphi-\varphi_{i}^{\prime}\right) / \sin \theta$. The notation $\alpha_{u, v}$ is used to indicate that we have to consider two odf's depending on whether $\omega_{i}^{\prime} \equiv \omega_{u, i}^{\prime}=\left(\varphi_{u, i}, \theta_{u, i}\right)$ or $\omega_{i}^{\prime} \equiv \omega_{v, i}^{\prime}=\left(\varphi_{v, i}, \theta_{v, i}\right)$ (see Figure 2).

There is an additional benefit of using the rotated coordinate systems. As we will demonstrate below the structure of the odf's turns out to be particularly simple in the rotated coordinate systems. For example, in a uniaxial nematic phase, $\alpha_{u}$ will exhibit two isolated maxima because of the reflection plane $\sigma_{\mathrm{h}}$ (see Figure 1); at the same time, $\alpha_{v}$ will have a band-like structure. In a biaxial nematic phase this band will change to a sequence of isolated maxima separated by a certain angle increment.

\subsection{Order parameters}

To classify various disordered and ordered phases in our system we now introduce order parameters via Equations (36) and (37). From the discussion in the preceding section we saw that the (instantaneous) eigensystem formed by the three vectors $\widehat{\boldsymbol{b}}_{1}, \widehat{\boldsymbol{b}}_{2}$, and $\widehat{\boldsymbol{b}}_{3}$ is shared by the three (instantaneous) tensors $\mathbf{Q}_{u u}, \mathbf{Q}_{v v}$, and $\mathbf{Q}_{w w}$. Moreover, by definition, $\widehat{\boldsymbol{b}}_{1}, \widehat{\boldsymbol{b}}_{2}$, and $\widehat{\boldsymbol{b}}_{3}$ define a rotating reference frame. This implies that during the course of a simulation none of these rotating frames is favoured over any other including that represented by the standard basis. This allows us to simplify the subsequent analysis by assuming that $\widehat{\boldsymbol{b}}_{1}=\widehat{\boldsymbol{e}}_{\mathrm{x}}, \widehat{\boldsymbol{b}}_{2}=\widehat{\boldsymbol{e}}_{\mathrm{y}}$, and $\widehat{\boldsymbol{b}}_{3}=\widehat{\boldsymbol{e}}_{\mathrm{z}}$ without loss of complete generality. 
We begin with the nematic order parameter defined as [cf., Ref. 45]

$$
S=\frac{1}{2} \int \mathrm{d} \Omega\left\{3\left[\widehat{\boldsymbol{u}}(\Omega) \cdot \widehat{\boldsymbol{b}}_{3}\right]^{2}-1\right\} \alpha(\Omega) .
$$

The integrand in this equation contains the scalar product $\left(\widehat{\boldsymbol{u}} \cdot \widehat{\boldsymbol{b}}_{3}\right)^{2}$ squared which reflects the existence of the reflection plane $\sigma_{\mathrm{h}}$ (see Figure 1). We can rewrite this expression using the tensor identity

$$
\left(\widehat{\boldsymbol{u}} \cdot \widehat{\boldsymbol{b}}_{3}\right)^{2}=\left[\left(\widehat{\boldsymbol{u}} \cdot \widehat{\boldsymbol{b}}_{3}\right) \widehat{\boldsymbol{u}}\right] \cdot \widehat{\boldsymbol{b}}_{3}=\widehat{\boldsymbol{b}}_{3} \cdot \widehat{\boldsymbol{u}} \widehat{\boldsymbol{u}} \cdot \widehat{\boldsymbol{b}}_{3}
$$

Noting that $1=\widehat{\boldsymbol{b}}_{3} \cdot \mathbf{1} \cdot \widehat{\boldsymbol{b}}_{3}$, we can rewrite Equation (42) as

$$
S=\int \mathrm{d} \Omega\left[\widehat{\boldsymbol{b}}_{3} \cdot \mathbf{Q}_{u u}^{\prime}(\Omega) \cdot \widehat{\boldsymbol{b}}_{3}\right] \alpha(\Omega),
$$

where [cf., Equation (36)]

$$
\mathbf{Q}_{u u}^{\prime}(\Omega)=\frac{1}{2}[3 \widehat{\boldsymbol{u}}(\Omega) \widehat{\boldsymbol{u}}(\Omega)-\mathbf{1}] .
$$

Alternatively, we may again start from Equation (42) and note that it may be rewritten as

$$
S=\int \mathrm{d} \Omega \mathcal{D}_{00}^{2}(\Omega) \alpha(\Omega)
$$

where we have used the fact that on account of our choice $\widehat{\boldsymbol{b}}_{3}=\widehat{\boldsymbol{e}}_{\mathrm{z}}$ the integrand can be cast as

$$
P_{2}(\cos \theta)=\frac{1}{2}\left(3 \cos ^{2} \theta-1\right)=\mathcal{D}_{00}^{2}(\Omega)
$$

where $\cos \theta=\widehat{\boldsymbol{u}} \cdot \widehat{\boldsymbol{b}}_{3}$. Using in Equation (46) the expansion of the odf introduced in Equation (31) we obtain

$$
S=\sum_{l m n} \alpha_{|m||n|}^{l} s_{|m||n|}^{\prime} \int \mathrm{d} \Omega \mathcal{D}_{00}^{2^{*}}(\Omega) \mathcal{D}_{m n}^{l}(\Omega),
$$

where $s_{m n}^{\prime}=s_{m n}(2 l+1) / 8 \pi^{2}$ and $s_{m n}$ is given in Equation (32). Invoking Equation (21) it is straightforward to verify that Equation (48) can be recast as

$$
S=\alpha_{00}^{2}
$$

It should be noted though that we included an extra factor of $(2 l+1) / 8 \pi^{2}$ in the definition of $s_{m n}^{\prime}$ to ensure that the expansion coefficients $\left\{\alpha_{|m||n|}^{l}\right\}$ become order parameters in the sense that the members of the set $\left\{\alpha_{|m||n|}^{l}\right\}$ take values in the interval $[0,1]$. We also note in passing that together the expressions given in Equations (46) and (47) agree with the one given by Camp and Allen [23] in their Equation (17), Equation (3.9) of Mulder [21], and Equation (5) in the paper by Straley [24]. 
Similarly, we can define the biaxiality order parameter through the expression

$$
\begin{aligned}
\eta \equiv & \frac{1}{2} \int \mathrm{d} \Omega\left[\left(\widehat{\boldsymbol{b}}_{1} \cdot \widehat{\boldsymbol{v}}\right)^{2}+\left(\widehat{\boldsymbol{b}}_{2} \cdot \widehat{\boldsymbol{w}}\right)^{2}\right. \\
& \left.-\left(\widehat{\boldsymbol{b}}_{2} \cdot \widehat{\boldsymbol{v}}\right)^{2}-\left(\widehat{\boldsymbol{b}}_{1} \cdot \widehat{\boldsymbol{w}}\right)^{2}\right] \alpha(\Omega) .
\end{aligned}
$$

As in Equation (42) the integrand in Equation (50) involves only (squared) scalar products between the vectors $\widehat{\boldsymbol{v}}$ and $\widehat{\boldsymbol{w}}$ and the two vectors $\widehat{\boldsymbol{b}}_{1}$ and $\widehat{\boldsymbol{b}}_{2}$ of the basis. As before this reflects the existence of the other two planes of reflection $\sigma_{\mathrm{wu}}$ and $\sigma_{\mathrm{vu}}$ as indicated by the cartoon in Figure 1. We can again take recourse to the tensor identity in Equation (43) and rewrite Equation (50) as

$$
\begin{aligned}
\eta= & \frac{1}{3} \int \mathrm{d} \Omega\left[\widehat{\boldsymbol{b}}_{1} \cdot \mathbf{Q}_{v v}^{\prime} \cdot \widehat{\boldsymbol{b}}_{1}+\widehat{\boldsymbol{b}}_{2} \cdot \mathbf{Q}_{w w}^{\prime} \cdot \widehat{\boldsymbol{b}}_{2}\right. \\
& \left.-\widehat{\boldsymbol{b}}_{2} \cdot \mathbf{Q}_{v v}^{\prime} \cdot \widehat{\boldsymbol{b}}_{2}-\widehat{\boldsymbol{b}}_{1} \cdot \mathbf{Q}_{w w}^{\prime} \cdot \widehat{\boldsymbol{b}}_{1}\right] \alpha(\Omega),
\end{aligned}
$$

where the tensors $\mathbf{Q}_{v v}^{\prime}$ and $\mathbf{Q}_{w w}^{\prime}$ are defined by complete analogy with the expression given in Equation (36). We also emphasise that the expression presented in Equation (51) agrees with Equation (19) in the paper of Camp and Allen [23].

That Equation (50) is a physically sensible definition of the biaxiality order parameter can be established as follows. Consider the case in which $\alpha$ is isotropic with respect to the axes $\widehat{\boldsymbol{b}}_{1}$ and $\widehat{\boldsymbol{b}}_{2}$. Let then $x$ be the cosine of the angles between each pair of the four vectors $\widehat{\boldsymbol{b}}_{1}$ and $\widehat{\boldsymbol{b}}_{2}$ on the one hand and $\widehat{\boldsymbol{v}}$ and $\widehat{\boldsymbol{w}}$ on the other hand. Thus, we are dealing with four integrals of the form $\pm \int_{-1}^{1} \mathrm{~d} x x^{2}= \pm \frac{2}{3}$ which cancel so that $\eta=0$ in the absence of biaxial order.

Notice also, that even if $\alpha$ is isotropic with respect to $\widehat{\boldsymbol{b}}_{1}$ and $\widehat{\boldsymbol{b}}_{2}$ it may or may not indicate alignment of the mesogens with respect to $\widehat{\boldsymbol{b}}_{3}$ so that $S=0$ or $S>0$. In the latter case, the $\mathcal{C}_{2}$-axis of the mesogens normal to the reflection plane $\sigma_{\mathrm{h}}$ (see Figure1) would be strongly aligned while the mesogens can freely rotate about this axis. If they can no longer rotate freely about the $\mathcal{C}_{2}$-axis we have to anticipate that $S>0$ and $\eta>0$.

We are now seeking to establish an alternative expression for $\eta$ in terms of the Wigner rotation matrices. To that end we remind the reader that because the mesogens are centrosymmetric only even integers $l, m$, and $n$ can arise. We also need to keep in mind that we are deliberately neglecting any coupling between uniaxial and biaxial degrees of freedom in the intermolecular interaction potential [see Equation (14)]. As a consequence and according to Mulder "... a spatially homogeneous phase will have a symmetry higher or equal to that of the constituent particles ..." [21]. By that token, $\alpha_{20}^{2}=\alpha_{02}^{2}=0$ because these expansion coefficients refer to a coupling between uniaxial and biaxial degrees of freedom [see Equations (A11) and (A12)]. We verified that in our simulations this expectation is met.

Moreover, invoking the same symmetry considerations delineated in Section 2, it seems obvious that we can alternatively consider

$$
\begin{aligned}
\eta & =\frac{1}{2} \int \mathrm{d} \Omega \mathcal{D}_{ \pm 2 \pm 2}^{2}(\Omega) \alpha(\Omega) \\
& =\frac{1}{2} \sum_{l m n} \alpha_{|m||n|}^{l} s_{|m||n|}^{\prime} \int \mathrm{d} \Omega \mathcal{D}_{ \pm 2 \pm 2}^{2 *}(\Omega) \mathcal{D}_{m n}^{l}(\Omega)
\end{aligned}
$$


where we employed Equation (31) and used $\mathcal{D}_{ \pm 2 \pm 2}^{2}$ as shorthand notation for the expression $\mathcal{D}_{22}^{2}+\mathcal{D}_{2 \underline{2}}^{2}+\mathcal{D}_{\underline{2} 2}^{2}+\mathcal{D}_{\underline{22}}^{2}$. Because of this definition $\mathcal{D}_{ \pm 2 \pm 2}^{2}$ is real. Invoking now the orthogonality of the Wigner rotation matrices [see Equation (21)] we obtain

$$
\eta=\frac{1}{2} \sum_{l m n} \frac{8 \pi^{2}}{2 l+1} \alpha_{|m||n|}^{l} s_{|m||n|}^{\prime} \delta_{l 2} \delta_{m \pm 2} \delta_{n \pm 2}
$$

which gives rise to four equivalent terms. Thus, using also Equation (32) together with the definition of $s_{m n}^{\prime}$ (see above) we arrive at our final result

$$
\eta=\alpha_{22}^{2}
$$

Finally, using the definition of the Wigner rotation matrices given in Equation (5) it is relatively straightforward to show that

$$
\begin{aligned}
\mathcal{D}_{ \pm 2 \pm 2}^{2}(\Omega)= & \left(\cos ^{2} \theta+1\right) \cos (2 \phi) \cos (2 \chi) \\
& -2 \cos \theta \sin (2 \phi) \sin (2 \chi) .
\end{aligned}
$$

Thus, using this result in Equation (52) we obtain

$$
\begin{aligned}
\eta= & \int \mathrm{d} \Omega\left[\frac{1}{2}\left(\cos ^{2} \theta+1\right) \cos (2 \phi) \cos (2 \chi)\right. \\
& -\cos \theta \sin (2 \phi) \sin (2 \chi)] \alpha(\Omega)
\end{aligned}
$$

which agrees with the expression presented in Equation (17) in the paper by Camp and Allen [23] and also matches Equation (3.9) in Mulder's paper [21] as well as Equation (5) in the paper by Straley [24].

\section{Results}

Throughout this work all physical quantities will be given in the customary dimensionless (i.e., "reduced") units. A comprehensive compilation of dimensionless units can be found in Appendix B of the book by Allen and Tildesley [46]. The results presented below have been obtained in Monte Carlo (MC) simulations carried out in the isothermal-isobaric ensemble. In this ensemble one generates a Markov chain with a limiting distribution in configuration space proportional to $\exp \left\{-\beta\left[U+P V-N \beta^{-1} \ln V\right]\right\}$ where $U$ is the total configurational potential energy, $P$ denotes the pressure, and $V$ is the volume occupied by the $N=5000$ mesogens.

Throughout this work, $P=1.00$. Our simulation runs comprise $10^{5} \mathrm{MC}$ steps consisting of $N$ random rotations or displacements of the mesogens followed by one attempt to change $V$. The decision whether a mesogen's centre of mass is to be displaced or whether it is rotated about one of its three symmetry axes is decided at random with equal on-average probability. Whether or not any of these attempts is accepted is decided on the basis of a modified Metropolis criterion such that the aforementioned limiting distribution in configuration space will eventually be reached. For details the interested reader is referred to standard textbooks in the field [46, 47]. 


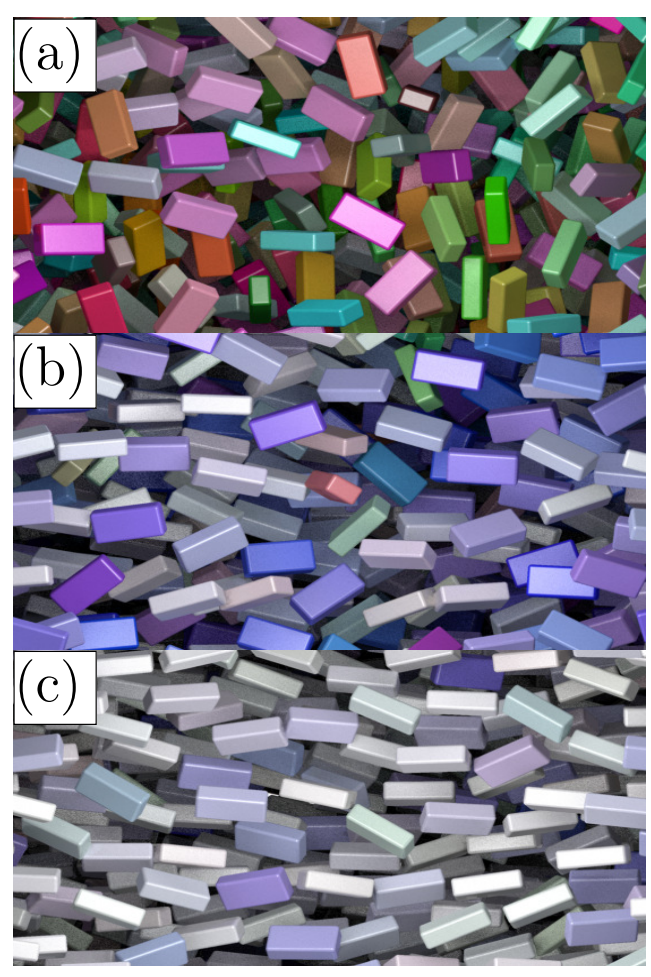

Figure 3. "Snapshots" of individual configurations obtained during the course of an MC simulation in the isothermal isobaric ensemble. (a) Isotropic phase, $T=1.120$; (b) uniaxial nematic phase, $T=1.030$; (c) biaxial nematic phase, $T=0.894$. The snapshots have been obtained for $\varepsilon^{\prime \prime}=0.048$. See text for an explanation of the colour code (see also Sections 2 and 4.1).

\subsection{The formation of uniaxial and biaxial nematic phases}

We begin the presentation of our results in Figure 3 by presenting "snapshots" of configurations obtained from our MC simulations. We remind the reader that in reality we are dealing with spherical particles (see discussion in Section 2) where the interaction potential is only equipped with certain symmetry elements corresponding to molecules that pertain to the point group $\mathrm{D}_{2 \mathrm{~h}}$. Matchboxes are just one of several possible realisations of objects belonging to that point group. To enhance the visualization of our simulation-generated data we arbitrarily assigned the shape of matchboxes to the mesogens under study.

The mesogens are coloured according to the following algorithm. Consider the three largest eigenvalues $\lambda_{+}^{u}, \lambda_{+}^{v}$, and $\lambda_{+}^{w}$ of the three tensors $\mathbf{Q}_{u u}, \mathbf{Q}_{v v}$, and $\mathbf{Q}_{w w}$ obtained from Equation (37). These eigenvalues are sorted such that $\mu_{+}=\max \left(\lambda_{+}^{u}, \lambda_{+}^{v}, \lambda_{+}^{w}\right)$ and $\mu_{-}=\min \left(\lambda_{+}^{u}, \lambda_{+}^{v}, \lambda_{+}^{w}\right)$; the remaining eigenvalue $\mu_{0}$ is then taken to be equal to that eigenvalue in the triplet $\lambda_{+}^{u}, \lambda_{+}^{v}$, and $\lambda_{+}^{w}$ that is smaller than $\mu_{+}$but larger than $\mu_{-}$. The eigenvectors associated with $\mu_{+}, \mu_{0}$, and $\mu_{-}$are given by $\widehat{\boldsymbol{n}}_{+}, \widehat{\boldsymbol{n}}_{0}$, and $\widehat{\boldsymbol{n}}_{-}$, respectively. They are also obtained as solutions of Equation (37).

We then introduce a triplet of real numbers $b_{i}=\widehat{\boldsymbol{u}}_{i} \cdot \widehat{\boldsymbol{n}}_{+}, r_{i}=\widehat{\boldsymbol{v}}_{\boldsymbol{i}} \cdot \widehat{\boldsymbol{n}}_{-}$, and $g_{i}=\widehat{\boldsymbol{w}}_{\boldsymbol{i}} \cdot \widehat{\boldsymbol{n}}_{0}$. The triplet $\left(r_{i}, g_{i}, b_{i}\right)$ defines a point in the red, green, and blue (rgb) colour space. Clearly, if the system is isotropic the mesogens appear in almost every colour of the $\mathrm{rgb}$ colour space. This is the case in Figure 3(a). In a uniaxial nematic phase, $b_{i}$ is largest whereas $r_{i}$ and $g_{i}$ are nearly zero. As a result the mesogens are predominantly coloured in shades of blue. This is the situation depicted in Figure 3(b). Finally, if 
the system is in the biaxial nematic phase $r_{i} \simeq g_{i} \simeq b_{i} \simeq 1.0$ which corresponds to a light grey or white colour. From Figure 3(c) we therefore conclude that this snapshot corresponds to a state of high biaxial order.

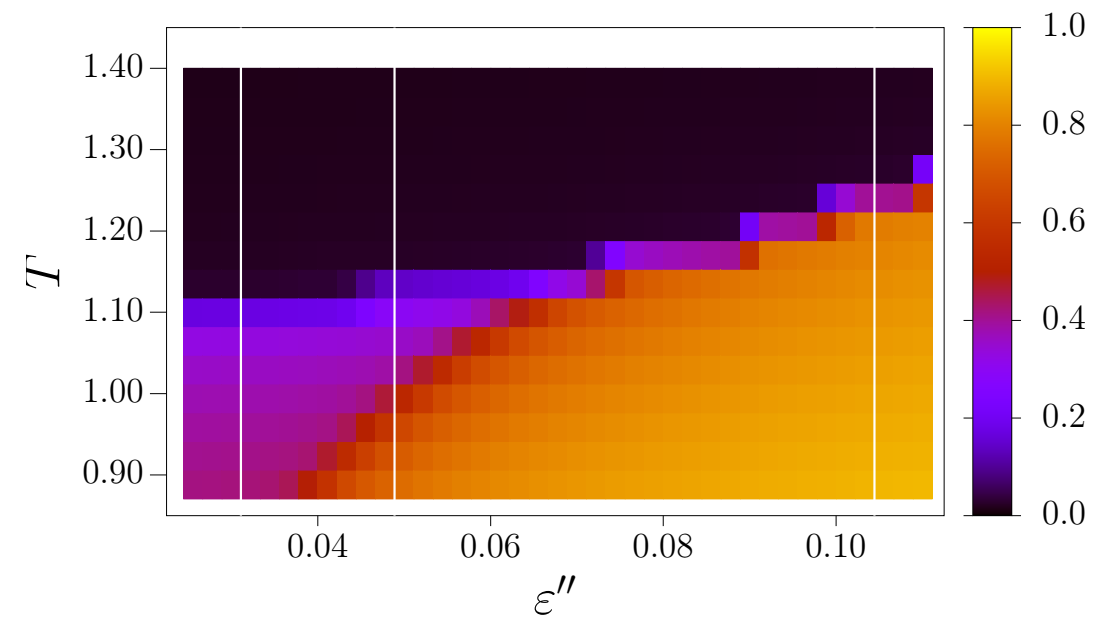

Figure 4. Plot of the arithmetic mean order parameter $\mathcal{O}$ (defined in the text) [see Equations (42) and (50)] in the plane spanned by the temperature $T$ and the dimensionless biaxial coupling constant $\varepsilon^{\prime \prime}$ [see Equation (14)]. The value of $\mathcal{O}$ can be read off the attached colour bar. The white vertical lines correspond to temperature scans for fixed biaxial coupling constants $\varepsilon^{\prime \prime}=0.03$ [see Figure $5(\mathrm{a})$ ], $\varepsilon^{\prime \prime}=0.05$ [see Figure $5(\mathrm{~b})$ ], and $\varepsilon^{\prime \prime}=0.10$ [see Figure 5(c).

To make this analysis more quantitative we consider in Figure 4 the order parameter $\mathcal{O}=\frac{1}{2}(S+\eta)$ corresponding to the arithmetic mean of the uniaxial and biaxial order parameters. On account of its definition, $\mathcal{O}=0$ in the isotropic liquid phase. In a perfectly ordered, uniaxial nematic phase $\mathcal{O}=0.5$ whereas $0.5 \leq \mathcal{O} \leq 1.0$ if the nematic phases possesses a certain degree of biaxial order. This is because in the biaxial nematic phase both $S$ and $\eta$ are both fairly large.

The plot in Figure 4 shows that regardless of the value of $\varepsilon^{\prime \prime}$ only an isotropic liquid phase is thermodynamically stable if the temperature $T$ is sufficiently high. As $T$ is lowered the mesogens begin to form a nematic phase which is uniaxial at first but then quickly becomes biaxial to a certain degree. The degree of biaxiality increases as $T$ is lowered according to ones physical expectation.

As $\varepsilon^{\prime \prime}$ decreases the temperature of the onset of order decreases as well. This is plausible because the formation of order is foiled by the kinetic energy the mesogens possess on average. For sufficiently low values $\varepsilon^{\prime \prime} \lesssim 0.04$ it seems that even at the lowest $T$ considered, biaxial order is completely absent or at least very small. The overall topology of the phase diagram plotted in Figure 4 matches qualitatively the one obtained by Sonnet et al. [5] at mean-field level (see their Figure 4).

These general features are illustrated in greater detail by the plots in Figure 5. For a relatively small biaxial coupling constant $\varepsilon^{\prime \prime}=0.03$ the plots in Figure 5(a) show that as $T$ is lowered, both $S$ and $\eta$ are nearly zero as one would expect for an isotropic liquid. At about $T \simeq 1.10, S$ increases rather abruptly and then keeps increasing slightly and monotonically until $T \simeq 0.80$ is reached. Because $\eta \simeq 0$ almost all the way down to $T \simeq 0.80$, the increase of $S$ at about $T \simeq 1.10$ signals the formation of a uniaxial nematic phase. We cannot go to significantly lower temperatures $T \lesssim 0.80$ because of the onset of a formation of glassy phases.

For a somewhat larger biaxial coupling constant $\varepsilon^{\prime \prime}=0.05$ we see from Figure $5(\mathrm{~b})$ that we have two characteristic temperatures instead of just one. At both temperatures 


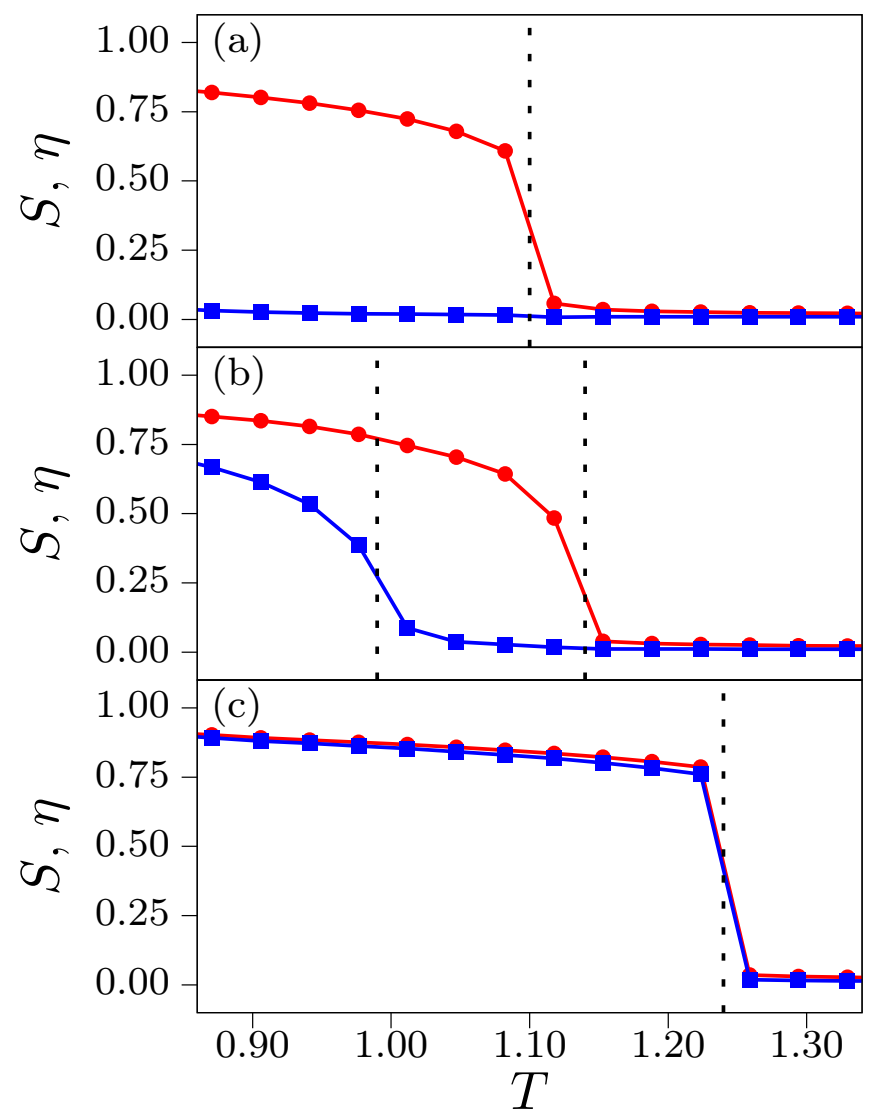

Figure 5. Plots of the nematic order parameter $S(\bullet)$ and of the biaxial order parameter $\eta(\square)$ as functions of the temperature $T$ for $(\mathrm{a}) \varepsilon^{\prime \prime}=0.03$, (b) $\varepsilon^{\prime \prime}=0.05$, and (c) $\varepsilon^{\prime \prime}=0.10$ (see Figure 4). The solid lines are intended to guide the eye and the vertical dashed lines demarcate the estimated (a) isotropic-uniaxial nematic $(T \simeq 1.10),(\mathrm{b})$ isotropic-uniaxial nematic $(T \simeq 1.14)$ and uniaxial-biaxial nematic $(T \simeq 0.99)$, and $(\mathrm{c})$ isotropic-biaxial nematic phase transitions $(T \simeq 1.24)$.

the order parameters increase markedly and independently. At the higher $T \simeq 1.14$, $S$ increases notably whereas $\eta$ remains practically zero. Thus, at this temperature the system undergoes a phase transition from an isotropic liquid to a uniaxial nematic phase. As $T$ is lowered further, $S$ keeps increasing monotonically indicating that the uniaxial nematic phase still becomes more ordered. At an even lower $T \simeq 1.00$, the biaxial order parameter $\eta$ starts rising as well. This indicates that at this $T$ we have a transition from a uniaxial to a biaxial nematic phase.

Finally, for the largest biaxial coupling constant $\varepsilon^{\prime \prime}=0.10$ the plots in Figure 5(c) reveal that we have only a single transition during which $S$ and $\eta$ simultaneously rise from about zero to fairly substantial values of $S \simeq \eta \simeq 0.75$. Thus, at the characteristic temperature $T \simeq 1.24$ at which this happens we observe a transition directly from an isotropic liquid to a biaxial nematic without an intermittent uniaxial nematic phase.

Notice also that the isotropic-biaxial nematic transition in Figure 5(c) occurs at the highest temperature compared with Figures 5(a) and 5(b). This is in line with one's physical intuition because the formation of a biaxial nematic is controlled by the largest biaxial coupling constant considered in the three parts of Figure 5. Therefore ordered phases may form even though the thermal energy of the mesogens is largest. 
(a)

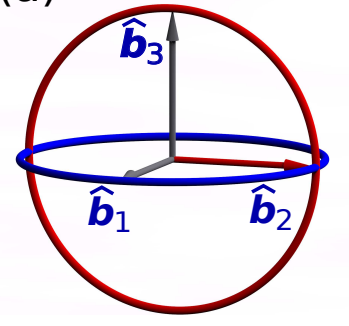

(b)

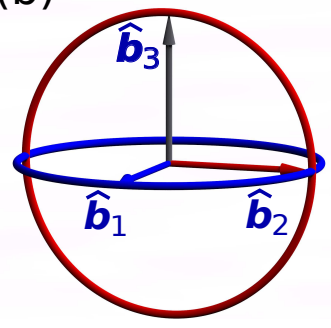

Figure 6. Sketch of the equatorial planes introduced in Section 3.2 to promote the analysis of the orientation distribution functions $\alpha_{u}$ and $\alpha_{v}$. The colour code is the same as the one introduced in Figure 2. The rings represent the respective equators in which $\alpha_{u}$ and $\alpha_{v}$ are computed after the rotations of the eigensystem described in Section 3.2. Arrows in the equatorial planes indicate preferred orientations of the mesogens if the system exhibits perfect uniaxial (a) or biaxial order (b).

\subsection{Orientational and positional structure}

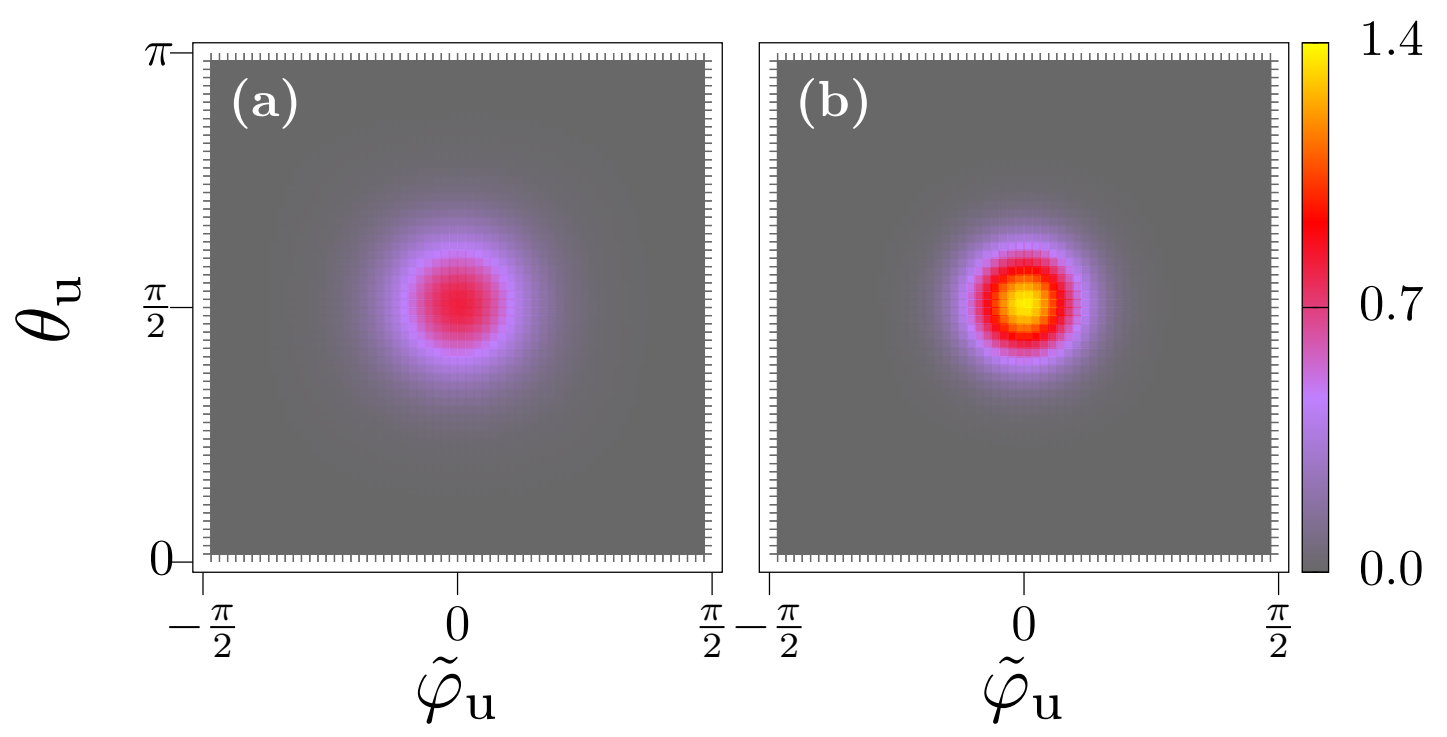

Figure 7. Plots of the orientation distribution function $\alpha_{u}$ in the $\widetilde{\varphi}_{u}-\theta_{u}$ plane where $\widetilde{\varphi}_{u}=\varphi_{u}-\pi\left(\varphi_{u} \in\right.$ $[0,2 \pi])$ has been shifted to center the plots on $\widetilde{\varphi}=0$. The value of $\alpha_{u}$ can be read off the attached colour bar. In all cases $\varepsilon^{\prime \prime}=0.048$; (a) $T=1.04$, (b) $T=0.91$.

After focusing on the formation of uniaxial and biaxial nematic phases in the preceding section we now elucidate details of the structure of these phases. Before showing any results we find it useful to remind the reader that we compute the odf's not with the original set of vectors $\left\{\widehat{\boldsymbol{u}}_{i}\right\}$ and $\left\{\widehat{\boldsymbol{v}}_{i}\right\}$ but with the rotated vectors $\left\{\widehat{\boldsymbol{u}}_{i}^{\prime}\right\}$ and $\left\{\widehat{\boldsymbol{v}}_{i}^{\prime}\right\}$ instead. The rotated vectors are obtained from Equations (38) as explained in Section 4. Hence, vectors of the sets $\left\{\widehat{\boldsymbol{u}}_{i}^{\prime}\right\}$ and $\left\{\widehat{\boldsymbol{v}}_{i}^{\prime}\right\}$ lie in their respective equatorial planes.

In a perfectly ordered uniaxial nematic phase all vectors of the set $\left\{\widehat{\boldsymbol{u}}_{i}^{\prime}\right\}$ are pointing in the direction of the axis $\pm \widehat{\boldsymbol{b}}_{2}$ of the equatorial plane represented by the red ring in Figure 6(a). Because the perfect nematic phase is uniaxial the vectors of the set $\left\{\widehat{\boldsymbol{v}}_{i}^{\prime}\right\}$ are distributed uniformly along the equatorial plane represented by the blue ring in Figure 6(a). Consequently, in a uniaxial nematic phase that is not perfectly ordered one would expect $\alpha_{u}$ to be centered on the axis labelled $\widehat{\boldsymbol{b}}_{2}$ in Figure $6(\mathrm{a})$ and represented by the red arrow. The corresponding odf $\alpha_{v}$ would be uniform along the equator in the 
plane $\widehat{\boldsymbol{b}}_{1}-\widehat{\boldsymbol{b}}_{2}$ with a certain width in the $\pm \widehat{\boldsymbol{b}}_{3}$-direction.

In the perfectly ordered biaxial nematic phase, schematically represented by the plot in Figure 6(b), the situation remains unaltered as far as $\alpha_{u}$ is concerned. However, in a perfect biaxial nematic phase the vectors of the set $\left\{\widehat{\boldsymbol{v}}_{i}^{\prime}\right\}$ are all pointing along the $\pm \widehat{\boldsymbol{b}}_{1}$-axis which is chosen at random with an equal probability. For a biaxial phase that is not perfectly ordered one expects an odf consisting of four more or less sharp maxima in the $\widehat{\boldsymbol{b}}_{1}-\boldsymbol{b}_{2}$ plane separated by an angle of $\pi$.

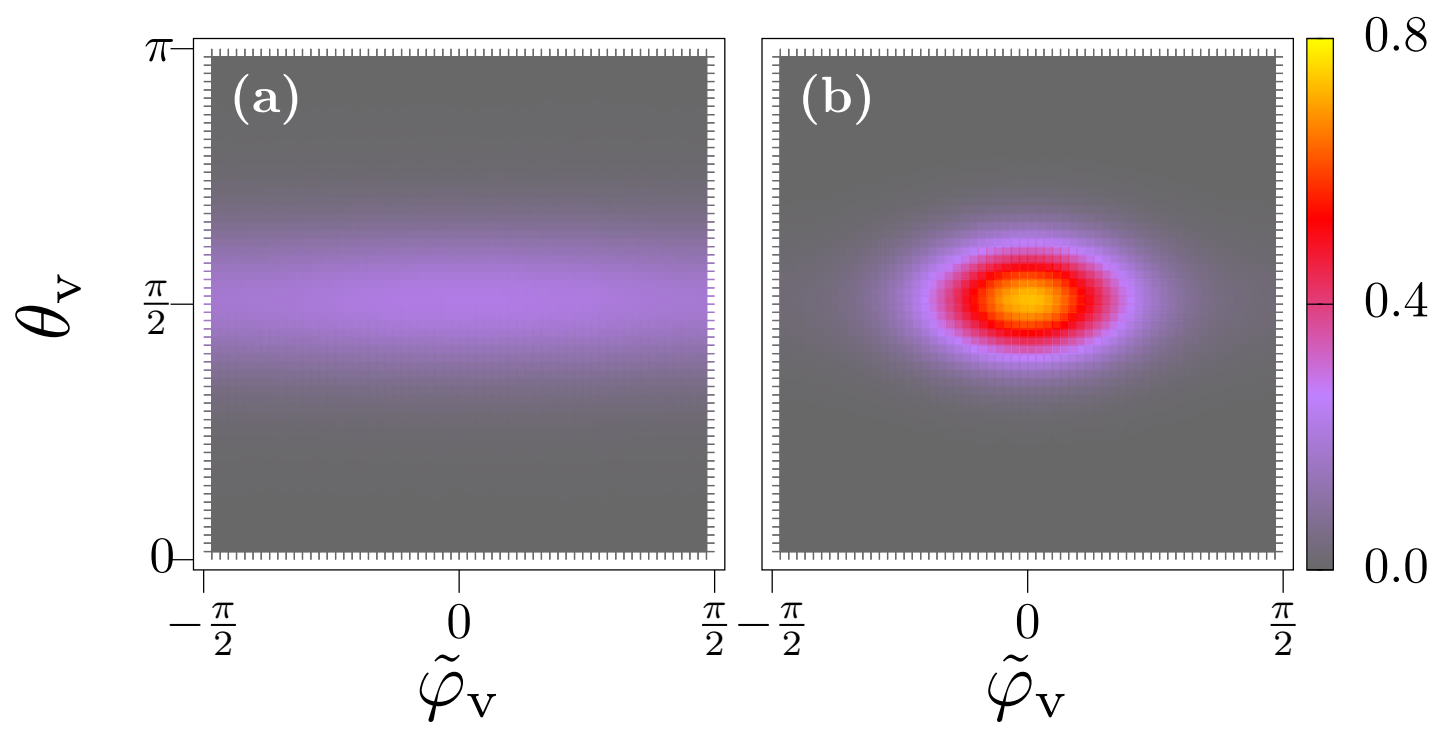

Figure 8. As Figure 7 but for $\alpha_{v}$.

We begin our discussion by presenting in Figure 7 plots of $\alpha_{u}$. We see that in both parts of the figure a single centrosymmetric spot appears that turns out to be higher at the lower $T$. The spots are centered on $\theta_{u}=\frac{\pi}{2}$ and $\widetilde{\varphi}_{u}=0$. To make contact with the cartoon shown in Figure 6(a) we see that the axis $\widehat{\boldsymbol{b}}_{1}$ should be orthogonal to the paper plane. We emphasise that $\alpha_{u}$ exhibits a second maximum at $\widetilde{\varphi}_{u}=\pi$ which we do not show in Figure 7. Because the spots in Figure 7 are centrosymmetric we can, however, exclude the existence of any extrinsic biaxiality.

We can also interpret the plot in Figure 7(a) in a slightly different way. It is clear that $\alpha_{u}$ reflects the distribution of the orientations $\left\{\widehat{\boldsymbol{u}}_{i}\right\}$ about the nematic director $\widehat{\boldsymbol{n}}$. Therefore, $\widehat{\boldsymbol{n}}$ is pointing in a direction normal to the paper plane. The centrosymmetric spot of the odf in Figure $7\left(\right.$ a) indicates that $\widehat{\boldsymbol{n}}$ is a $\mathcal{C}_{\infty}$ rotation axis.

Unfortunately, we cannot distinguish uniaxial from biaxial nematic phases on the basis of $\alpha_{u}$ alone which holds for the presence of intrinsic biaxiality. In order to discriminate between both symmetries we need to consider the odf calculated for a second symmetry axis. To that end we consider in Figure 8 plots of $\alpha_{v}$. The data plotted in Figure 8 (a) can be represented by a band-like structure of a certain width around $\theta_{v}=\frac{\pi}{2}$ extending along the $\widetilde{\varphi}_{v}$-axis. The existence of the band reflects the uniform distribution of $\left\{\widehat{\boldsymbol{v}}_{i}^{\prime}\right\}$ along the equatorial plane shown in Figure 6(a). The width of the band is a measure of the average deviation of the vectors $\left\{\widehat{\boldsymbol{v}}_{i}^{\prime}\right\}$ from the $\widehat{\boldsymbol{b}}_{3}$-axis. Thus, on account of the combined information contained in Figures 7(a) and 8(a) we conclude that the system is in the uniaxial nematic phase.

At the lower $T=0.91$ the corresponding plot presented in Figure 8(b) now exhibits a sequence of isolated, slightly elongated spots where the major axis is parallel to the 


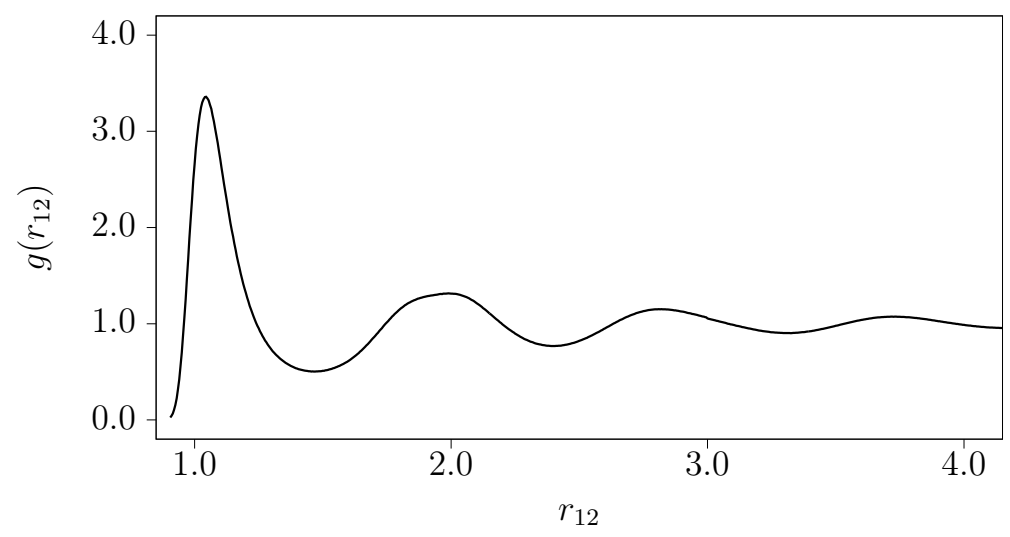

Figure 9. Plot of the radial pair correlation function $g\left(r_{12}\right)$ as a function of the centre-of-mass distance $r_{12}$ for a pair of mesogens at a temperature $T=0.89$ and $\varepsilon^{\prime \prime}=0.05$ at which the system is in the biaxial nematic phase (see Figure 4).

$\widetilde{\varphi}_{v}$-axis and the minor axis is parallel to the $\theta_{v}$-axis. Because of the arguments put forth when discussing the cartoon shown in Figure 6(b), these centres of the spots are separated by an angle increment of $\Delta \widetilde{\varphi}_{v}=\frac{\pi}{2}$. Thus, we conclude that the plots shown in Figures 7(b) and 8(b) are indicative of a biaxial nematic phase. Notice again that we are showing only one of the two maxima of which $\alpha_{v}$ consists in the biaxial nematic phase.

In other words, because the centrosymmetric character of the spot of $\alpha_{u}$ in Figure 7 (b) remains unchanged compared with Figure $7(\mathrm{a}), \widehat{\boldsymbol{n}}$ remains a $\mathcal{C}_{\infty}$ rotation axis even if the system is biaxial. By the same token, $\alpha_{v}$ plotted in Figure $8(\mathrm{~b})$ reflects the distribution of orientations of the mesogens about the secondary symmetry axis which is also pointing in a direction normal to the paper plane. However, the difference is that this secondary symmetry axis has $\mathcal{C}_{2}$ character unlike $\widehat{\boldsymbol{n}}$. We ascribe this to the lack of coupling between uniaxial and biaxial degrees of freedom in our model.

We now turn to an analysis of orientational and positional correlations in our system. To make sure that our system is still fluidic we consider the radial pair correlation function defined as [38]

$$
g\left(r_{12}\right)=\frac{1}{\left(8 \pi^{2}\right)^{2}} \iint \mathrm{d} \Omega_{1} \mathrm{~d} \Omega_{2} g\left(r_{12}, \Omega_{1}, \Omega_{2}\right) .
$$

A plot of this quantity is presented in Figure 9 for a thermodynamic state point at which the system is in a stable biaxial nematic phase. As one can see in Figure 9, spatial correlations are still short-range and decay within a couple of diameters $\sigma$ of the spherical core of our matchbox-symmetric mesogens. However, there is a noticeable shift of the maxima to progressively smaller values of $r_{12}$ that is untypical for a simple fluid. Whereas the first peak of $g$ appears at about the value at which $u_{\mathrm{LJ}}$ in Equation (13) passes through its minimum, higher-order peaks appear at non-integer multiples of the position of the first maximum. This feature is ascribed to the different (non-spherical) symmetry of the biaxial nematic phase. However, we emphasize that the curve shown in Figure 9 is free of any pathological features that would indicate an artificial (and unwanted) glassy structure.

We are now turning to the orientation correlation functions. These are accessible through Equations (33). Generally speaking, the orientation correlation functions plot- 


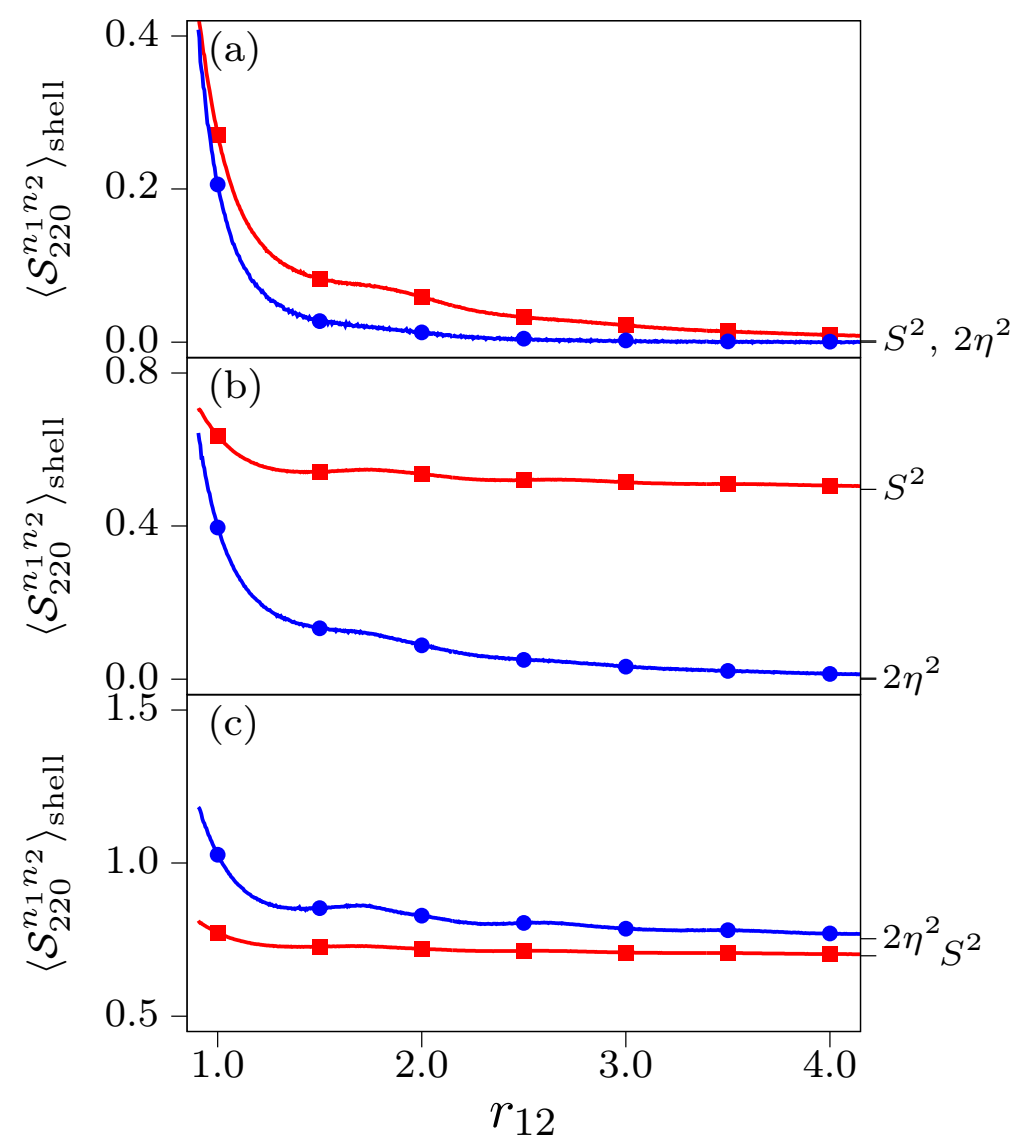

Figure 10. Plots of the orientation correlation functions $\left\langle\mathcal{S}_{220}^{n_{1} n_{2}}\right\rangle_{\text {shell }}$ as functions of the distance $r_{12}$ between a pair of mesogens; $(\boldsymbol{\square}) n_{1}=n_{2}=0,(\bullet) n_{1}= \pm 2, n_{2}= \pm 2$. In all parts of the figure, $\varepsilon^{\prime \prime}=0.05 ;$ (a) $T=1.12$, (b) $T=1.03$, (c) $T=0.89$. Also indicated are the limiting values of $S^{2}$ and $2 \eta^{2}$ obtained from Equations (42) and (50). Note that in all three parts of the figure, the limiting values $S^{2}$ and $2 \eta^{2}$ are approached from above as $r_{12}$ increases according to one's physical intuition.

ted in the three parts of Figure 10 exhibit far less structure than the radial pair correlation function shown in Figure 9. In the isotropic phase, the plots presented in Figure 10(a) indicate that $\left\langle\mathcal{S}_{220}^{n_{1} n_{2}}\right\rangle_{\text {shell }}$ decay monotonically and vanish completely within a couple of diameters $\sigma$ of the spherical cores. It turns out that $\left\langle\mathcal{S}_{220}^{ \pm 2 \pm 2}\right\rangle_{\text {shell }}$ vanishes more rapidly than $\left\langle\mathcal{S}_{220}^{00}\right\rangle_{\text {shell }}$ indicating that biaxial short-range order is lost earlier.

The data shown in Figure 10(b) pertain to a state point at which the system is in a uniaxial nematic phase. This is concluded because $\left\langle\mathcal{S}_{220}^{ \pm 2 \pm 2}\right\rangle_{\text {shell }}$ is still short-range and vanishes on a molecular length scale whereas $\left\langle\mathcal{S}_{220}^{00}\right\rangle_{\text {shell }}$ has become long-range with no appreciable tendency to decay towards zero.

Finally, in the biaxial nematic phase $\left\langle\mathcal{S}_{220}^{00}\right\rangle_{\text {shell }}$ as well as $\left\langle\mathcal{S}_{220}^{ \pm 2 \pm 2}\right\rangle_{\text {shell }}$ exhibit longrange order as one might have expected. However, it is particularly gratifying that in all three parts of Figure 10 the curves decay to the values predicted by Equations (35a) and (35b). This lends credibility to our simulations because the limiting values of $S^{2}$ and $2 \eta^{2}$ were computed independently from $S$ and $\eta$ as given by Equations (42) and (50).

In view of the results just discussed it seems instructive to focus on the decay of 


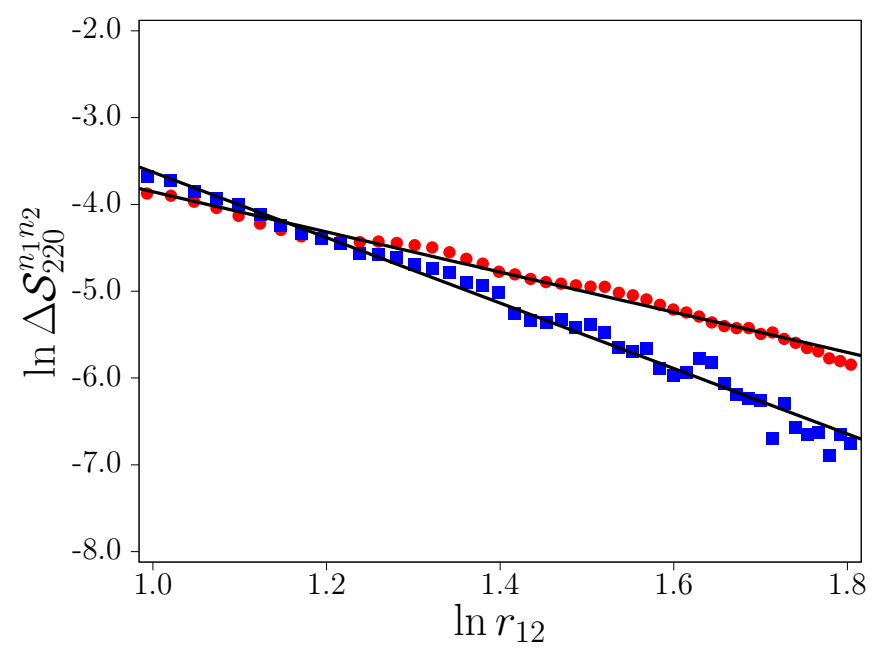

Figure 11. Double-logarithmic plots of $\Delta \mathcal{S}_{220}^{n_{1} n_{2}}$ as functions of $r_{12} ;(\boldsymbol{\bullet}) n_{1}=n_{2}=0$, (ם) $n_{1}= \pm 2$, $n_{2}= \pm 2$. The straight fines are linear fits to the discrete data points. The data shown correspond to $T=1.08$ and $\varepsilon^{\prime \prime}=0.05$

short-range orientational correlations. To that end we consider

$$
\begin{aligned}
\Delta \mathcal{S}_{220}^{00} & \equiv\left\langle\mathcal{S}_{220}^{00}\right\rangle_{\text {shell }}-S^{2} \\
\Delta \mathcal{S}_{220}^{ \pm 2 \pm 2} & \equiv\left\langle\mathcal{S}_{220}^{ \pm 2 \pm 2}\right\rangle_{\text {shell }}-2 \eta^{2}
\end{aligned}
$$

which vanish by definition in the limit of $r_{12} \rightarrow \infty$. Moreover, let us assume that both quantities defined in Equations (58) decay exponentially. Thus, we can define a correlation length $\xi_{n_{1} n_{2}}$ as the slope of the straight lines shown in Figure 11.

Repeating the analysis illustrated by the plots in Figure 11 for different temperatures we obtain the curves shown in Figure 12. The plots in Figure 12(a) show that $\xi_{00}$ decays towards the temperature at which a uniaxial nematic phase forms; it assumes a constant value of about 3.0 for all lower $T$. The plot of $\xi_{ \pm 2 \pm 2}$ increases monotonically throughout the temperature range considered. This correlates nicely with the absence of a uniaxial-biaxial nematic transition but a steady increase of short-range biaxial correlations in the corresponding Figure 5(a). It seems plausible that $\xi_{00}$ assumes a constant value once the uniaxial nematic phase has formed because of our definition of the correlation lengths.

Similarly, the plots in Figure 12(b) show very similar trends. Towards the isotropicuniaxial nematic transition, $\xi_{00}$ decays and then assumes a constant value for all lower $T$. On the contrary, $\xi_{ \pm 2 \pm 2}$ increases towards the uniaxial-biaxial nematic transition and then decays again for all lower $T$. Notice, in particular, that below the uniaxialbiaxial-nematic transition $\xi_{00} \approx \xi_{ \pm 2 \pm 2}$. The latter effect is seen more clearly in the plots presented in Figure 12(c) where no noticeable variation of $\xi_{00}$ and $\xi_{ \pm 2 \pm 2}$ is detected by lowering $T$ below the isotropic-biaxial nematic transition temperature at $T \simeq 1.24$.

\section{Discussion and conclusions}

By means of MC simulations in the isothermal-isobaric ensemble we investigate the formation and properties of uniaxial and biaxial nematic phases in a thermotropic liquid crystal. The liquid crystal is composed of matchbox-symmetric mesogens which 


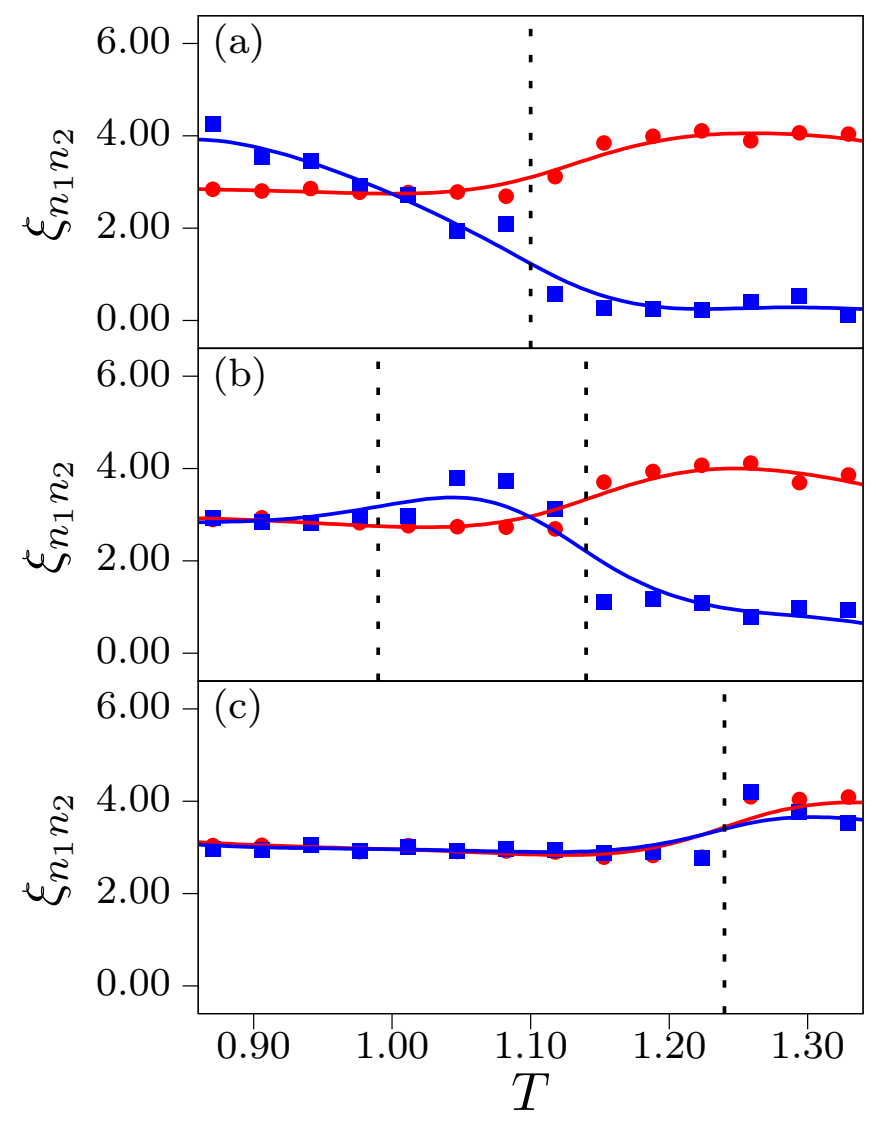

Figure 12. Plots of the correlation length $\xi_{n_{1} n_{2}}$ (see text) as a function of the temperature $T$ for $n_{1}=n_{2}=0$ ( ) and $n_{1}= \pm 2, n_{2}= \pm 2(\boldsymbol{\square})$. Solid lines are fits to the discrete data points intended to guide the eye; (a) $\varepsilon^{\prime \prime}=0.03$, (b) $\varepsilon^{\prime \prime}=0.05$, (c) $\varepsilon^{\prime \prime}=0.10$. Vertical dashed lines indicate the temperatures at which ordered phases form (cf., Figure 5).

possess an intrinsic biaxiality. This is reflected by the fact that the mesogens have a major and two equivalent minor symmetry axes and thus possess three mutually orthogonal reflection planes.

In a number of recent computer simulation studies $[28,48-50]$ the formation of ordered phases was studied for models in which the molecular constituents interact via purely hard repulsive potentials. In such systems it turns out to be a bit difficult to form and stabilise ordered structures. This could be because of the purely entropic nature of the interactions between the particles which may be too weak. In addition, any attempt to change the orientation of the molecules during the course of a simulation is difficult to accomplish. One way to overcome this difficulty is to use external fields [50]. Another disadvantage of such model systems in computer simulations is that the interaction strength cannot be controlled easily.

We are proposing a different strategy in this work. Rather than modeling the shape of the mesogens explicitly we take spherical molecules and endow them with an orientation-dependent attraction that accounts for the symmetry elements of molecules of the desired point group. This philosophy follows in spirit the earlier work of Maier and Saupe [41]. Thus, in a sense we are dealing with pseudo-shaped mesogens.

Systems composed of pseudo-shaped mesogens generally allow for an equilibration that is orders of magnitude faster than for models with explicit shape dependence. At the same time pseudo-shaped mesogens do exhibit physically sensible properties 
as we demonstrated in a number of earlier publications [51-53]. From our point of view purely entropic interactions have quite a few shortcomings as far as simulation studies are concerned. However, we stress that their use can be quite advantageous in theoretical approaches such as classical density functional theory [54] on account of the vanishing range of the entropic interactions.

In this work the interaction between a pair of mesogens is described by a model potential where the orientation dependence is the same as that proposed originally by Luckhurst and Romano [30]. The model proposed by these authors has also been used later by Longa and Pająk [31]. In the Luckhurst-Romano model the orientation dependence of the interactions follows from an expansion of the potential in terms of Stone's rotational invariants $\left\{\mathcal{S}_{l_{1} l_{2} l}^{n_{1} n_{2}}\right\}$ [29]. These functions are rotationally invariant products of the Wigner rotation matrices $\mathcal{D}_{m n}^{l}[38,39]$. However, we are using an off-lattice model unlike the Luckhurst-Romano model.

We emphasise that the expansion in Wigner rotation matrices of the interaction potential and of the orientation-dependent pair correlation function can also be cast in terms of the contraction of Cartesian tensors. These tensors describe the coupling between uniaxial degrees of freedom on the one hand and biaxial degrees of freedom on the other hand. The latter formulation is identical with the one proposed by Sonnet et al. [5] and by Sonnet and Virga [36]. We have verified here that both formulations are indeed the same. The proof of this equivalence rests upon a ladder-operator algebra [34] borrowed from the quantum-mechanical theory of angular momentum [35].

In summary, we are presenting three key results in this work. The first of these concerns the good agreement of the topology of our phase diagram (see Figure 4) with the one presented by Sonnet et al. [5] and obtained within their mean-field treatment. Within the parameter space investigated here three types of phase transitions can occur. The first of these exhibits only an isotropic to uniaxial nematic transition. The second one allows for a sequence of separate isotropic-uniaxial nematic followed by a uniaxial-biaxial nematic transition. The third scenario takes one directly from an isotropic to a biaxial nematic phase. Relatively recent experimental evidence for the latter two scenarios seems to be provided by ${ }^{13} \mathrm{C}$ NMR spectroscopy [13]. Theoretically, the above sequence of phase transitions is predicted by the phenomenological GinzburgLandau theory [31].

The second key result concerns an analysis of the odf. From a separate analysis of the odf's referring to the respective major and (one of the two) minor symmetry axes we can unambiguously distinguish between uniaxial nematic and biaxial nematic orientational structures. At its core, the analysis is enabled by properly rotating the molecular major and minor symmetry axes such that they always lie in the equatorial plane of the unit sphere. This way we are able to combine an enhanced resolution of the odf with a maximum statistical accuracy with which the odf's are accessible in a computer simulation. The idea to employ a rotating rather than a fixed reference frame has been proposed in the work of Skutnik et al. [7].

On the basis of the odf one can unambiguously distinguish between a system with intrinsic and one with extrinsic biaxiality. For the latter it was recently demonstrated [7] that in a binary mixture of two uniaxial components a globally biaxial nematic phase can form if the orientation dependence of the interaction between two unlike mesogens is tuned such that a T-shaped arrangement is energetically favoured. In the biaxial phase the odf is elliptically deformed in the $\widetilde{\varphi}-\theta$ plane where the two major axes of the ellipses are orthogonal to one another. In a system with extrinsic biaxiality only one odf conveys the entire information about the structure of the biaxial nematic phase. 
Because of the reflection plane $\sigma_{\mathrm{h}}$ the $\alpha_{u}$ exhibits two equivalent circular maxima that are separated by an angle increment $\Delta \widetilde{\varphi}_{u}=\pi$ regardless of whether one is in the uniaxial or biaxial nematic phase.

In the uniaxial nematic phase $\alpha_{v}$ consists of a strip centered on $\theta_{u}=\frac{\pi}{2}$ because there is no particular order with respect to the two minor symmetry axes. In the biaxial nematic phase, the reflection plane $\sigma_{\mathrm{v}}^{w u}$ suggests that the odf $\alpha_{v}$ should also exhibit two maxima separated by an angle $\Delta \widetilde{\varphi}_{v}=\pi$. However, one observes two maxima that are but separated by $\Delta \widetilde{\varphi}_{v}=\pi$. This is because the two minor molecular axes $\widehat{\boldsymbol{v}}_{i}$ and $\widehat{\boldsymbol{w}}_{i}$ are degenerate because we neglect the coupling between uniaxial and biaxial degrees of freedom in our model. Thus, our mesogens pertain to the $\mathrm{D}_{2 \mathrm{~h}}$ point group.

The simple structure of the odf's reflects the overall symmetry of the interaction potential that does not contain a coupling between uniaxial and biaxial degrees of freedom. Therefore, the two maxima of $\alpha_{u}$ as well as the two maxima of $\alpha_{v}$ in the biaxial nematic phase have equal height. It is anticipated that when this symmetry is broken by allowing for this coupling, the centrosymmetric spots of $\alpha_{u}$ will be elliptically deformed as well. In other words, the $\mathcal{C}_{\infty}$ of $\widehat{\boldsymbol{n}}$ will presumably change to the lower $\mathcal{C}_{2}$ symmetry already seen in $\alpha_{v}$ in the present work.

The difference between that earlier work and the present one is that here we are employing as a reference system the system of eigenvectors of the three alignment tensors referring to the major and the two minor symmetry axes of the mesogens. This approach follows in spirit the one used by Allen [22] and by Camp and Allen [23] in their calculation of the uniaxial and biaxial order parameters. These authors did, however, make no attempt to analyse the odf.

The third key result is related to our analysis of the orientation-dependent pair correlation function when their expansion is made in terms of Stone's rotational invariants [29]. This expansion was proposed earlier by Blum and Torruella [55] who did, however, not show any actual data for the expansion coefficients. We demonstrate in this work that the expansion coefficients become long-range when an ordered phase has formed. As we show here the limiting values of the correlation functions in general involve a subtle coupling between different order parameters. The simpler result obtained quite some time ago by Zannoni $[37,43]$ is recovered as a special case of our treatment.

Based upon the assumption that the decay of the expansion coefficients towards their long-range value is exponential, we can extract a correlation length for the short-range correlations. This correlation length assumes a constant value once the "macroscopically" ordered phase has formed. For thermodynamic states prior to the formation of "macroscopically" ordered phases the temperature dependence of the correlation length signals uniaxial or biaxial "pre-ordering". The biaxial pre-ordering is a precursor of a biaxial nematic phase eventually triggering the latter. This situation is very much akin to that encountered experimentally where first tetrapodes are being synthesised that are of biaxial symmetry only on a local length scale. These then initiate the formation of a globally biaxial nematic phase given the right thermodynamic conditions [17].

Last but not least, we emphasise that the approach employed here is flexible enough to be extended to treat interaction potentials in models of bent-core mesogens. These mesogens have two rather than three reflection planes and thus a somewhat lower symmetry compared with the matchbox-symmetric mesogens studied here. In these systems undulated biaxial orientational order can emerge [see Figure 1(e) of Ref. 56]. Such undulated structures are conceivable if for the present model the uniaxial- and biaxial-tensor contributions are modified to allow for a certain handedness of the interaction potential in two directions. That way it seems conceivable that one could study systems exhibiting chiral $\mathrm{C}^{*}$ phases. Work along these lines is currently in progress. 


\section{Appendix A. Cartesian tensors}

The purpose of this Appendix is to derive expressions for $\mathcal{S}_{220}^{n_{1} n_{2}}$ arising in the expansion of $u_{\text {aniso }}$ in Equation (6). We begin the discussion with $\mathcal{S}_{220}^{00}$ expressing it as a Cartesian tensor according to [29]

$$
2 \sqrt{5} \mathcal{S}_{220}^{00}\left(\Omega_{1}, \Omega_{2}, \Omega_{12}\right)=3\left(\widehat{\boldsymbol{u}}_{1} \cdot \widehat{\boldsymbol{u}}_{2}\right)^{2}-1,
$$

where $\widehat{\boldsymbol{u}}_{i}$ is given in Equation (11a). Using the identity (see Section 2)

$$
\widehat{\boldsymbol{u}}_{1} \widehat{\boldsymbol{u}}_{1}: \widehat{\boldsymbol{u}}_{2} \widehat{\boldsymbol{u}}_{2}=\operatorname{Tr}\left(\widehat{\boldsymbol{u}}_{1} \widehat{\boldsymbol{u}}_{1} \cdot \widehat{\boldsymbol{u}}_{2} \widehat{\boldsymbol{u}}_{2}\right)=\left(\widehat{\boldsymbol{u}}_{1} \cdot \widehat{\boldsymbol{u}}_{2}\right)^{2}
$$

and the definition of $\mathbf{U}$ given in Equation (10a), it is a simple matter to verify that

$$
\mathcal{S}_{220}^{00}\left(\Omega_{1}, \Omega_{2}, \Omega_{12}\right)=\frac{1}{\sqrt{5}} \frac{3}{2} \mathbf{U}_{1}: \mathbf{U}_{2}
$$

To proceed it turns out to be most convenient [29] to draw upon the angularmomentum algebra in quantum mechanics [35] and introduce the differential operator

$$
\boldsymbol{L}_{j}=-i \nabla_{\Omega_{j}}
$$

such that [29]

$$
\boldsymbol{L}_{j}\left(\widehat{\boldsymbol{v}}_{j} \widehat{\boldsymbol{w}}_{j} \widehat{\boldsymbol{u}}_{j}\right)=\left(\begin{array}{ccc}
0 & -i \widehat{\boldsymbol{u}}_{j} & i \widehat{\boldsymbol{w}}_{j} \\
i \widehat{\boldsymbol{u}}_{j} & 0 & -i \widehat{\boldsymbol{v}}_{j} \\
-i \widehat{\boldsymbol{w}}_{j} & i \widehat{\boldsymbol{v}}_{j} & 0
\end{array}\right)
$$

Again by complete analogy with quantum mechanics we introduce the ladder operators

$$
L_{j \pm}=L_{j v} \pm i L_{j w}
$$

satisfying the equation

$$
\begin{aligned}
& L_{1 \pm} \mathcal{S}_{l_{1} l_{2} l}^{n_{1} n_{2}}\left(\Omega_{1}, \Omega_{2}, \Omega_{12}\right) \\
= & {\left[l_{1}\left(l_{1}+1\right)-n_{1}\left(n_{1} \mp 1\right)\right]^{1 / 2} \mathcal{S}_{l_{1} l_{2} l}^{\left(n_{1} \mp 1\right) n_{2}}\left(\Omega_{1}, \Omega_{2}, \Omega_{12}\right) . }
\end{aligned}
$$

The subscript $j$ refers to the mesogen the ladder operator is acting upon.

Applying Equation (A7) to $\mathcal{S}_{220}^{00}$ and using Table 2 of the paper by Stone [29] we obtain

$$
\begin{aligned}
L_{1+} \mathcal{S}_{220}^{00} & =\sqrt{6} \mathcal{S}_{220}^{10} \\
& =\frac{1}{2 \sqrt{5}}\left(L_{1 v}+i L_{1 w}\right)\left[3\left(\widehat{\boldsymbol{u}}_{1} \cdot \widehat{\boldsymbol{u}}_{2}\right)^{2}-1\right] \\
& =\frac{3}{\sqrt{5}}\left(\widehat{\boldsymbol{u}}_{1} \cdot \widehat{\boldsymbol{u}}_{2}\right)\left(\widehat{\boldsymbol{v}}_{1}+i \widehat{\boldsymbol{w}}_{1}\right) \cdot \widehat{\boldsymbol{u}}_{2}
\end{aligned}
$$

where we dropped the arguments of $\mathcal{S}_{220}^{00}$ and $\mathcal{S}_{220}^{10}$ for the time being and also used 
Equation (A5). From the previous expression it then follows that

$$
\mathcal{S}_{220}^{10}=\sqrt{\frac{3}{10}}\left(\widehat{\boldsymbol{u}}_{1} \cdot \widehat{\boldsymbol{u}}_{2}\right)\left(\widehat{\boldsymbol{v}}_{1}+i \widehat{\boldsymbol{w}}_{1}\right) \cdot \widehat{\boldsymbol{u}}_{2}
$$

Applying $L_{1+}$ one more time to this result we obtain

$$
\begin{aligned}
L_{1+} \mathcal{S}_{220}^{10} & =2 \mathcal{S}_{220}^{20} \\
& =\sqrt{\frac{3}{10}} L_{1+}\left(\widehat{\boldsymbol{u}}_{1} \cdot \widehat{\boldsymbol{u}}_{2}\right)\left(\widehat{\boldsymbol{v}}_{1}+i \widehat{\boldsymbol{w}}_{1}\right) \cdot \widehat{\boldsymbol{u}}_{2} \\
& =\sqrt{\frac{3}{10}}\left[\left(\widehat{\boldsymbol{v}}_{1}+i \widehat{\boldsymbol{w}}_{1}\right) \cdot \widehat{\boldsymbol{u}}_{2}\right]^{2} \\
& =\sqrt{\frac{3}{10}}\left[\left(\widehat{\boldsymbol{v}}_{1} \cdot \widehat{\boldsymbol{u}}_{2}\right)^{2}-\left(\widehat{\boldsymbol{w}}_{1} \cdot \widehat{\boldsymbol{u}}_{2}\right)^{2}+2 i\left(\widehat{\boldsymbol{v}}_{1} \cdot \widehat{\boldsymbol{u}}_{2}\right)\left(\widehat{\boldsymbol{w}}_{1} \cdot \widehat{\boldsymbol{u}}_{2}\right)\right] .
\end{aligned}
$$

We can finally solve this expression for $\mathcal{S}_{220}^{20}$ and obtain

$$
\mathcal{S}_{220}^{20}=\frac{1}{2} \sqrt{\frac{3}{10}}\left[\mathbf{B}_{1}: \mathbf{U}_{2}+2 i\left(\widehat{\boldsymbol{v}}_{1} \cdot \widehat{\boldsymbol{u}}_{2}\right)\left(\widehat{\boldsymbol{w}}_{1} \cdot \widehat{\boldsymbol{u}}_{2}\right)\right],
$$

where we used Equations (10a), (10b), (A2), and the fact that the tensors $\widehat{\boldsymbol{u}}_{2} \widehat{\boldsymbol{u}}_{2}, \widehat{\boldsymbol{v}}_{1} \widehat{\boldsymbol{v}}_{1}$, and $\widehat{\boldsymbol{w}}_{1} \widehat{\boldsymbol{w}}_{1}$ satisfy the distributive law. Notice also that we do not have to worry whether or not we have to take the transpose of any tensor arising in the above manipulations because they are all real and symmetric.

The derivation of $\mathcal{S}_{220}^{20}$ proceeds exactly the same way. The calculation can be shortened considerably by noting that in successive applications of $L_{1-}, L_{1-} \widehat{\boldsymbol{u}}_{1}=$ $-\left(\widehat{\boldsymbol{v}}_{1}-i \widehat{\boldsymbol{w}}_{1}\right)$ whereas $L_{1-}\left(\widehat{\boldsymbol{v}}_{1}-i \widehat{\boldsymbol{w}}_{1}\right)=0$. Thus, at the end of the day we obtain

$$
\mathcal{S}_{220}^{20}=\frac{1}{2} \sqrt{\frac{3}{10}}\left[\mathbf{B}_{1}: \mathbf{U}_{2}-2 i\left(\widehat{\boldsymbol{v}}_{1} \cdot \widehat{\boldsymbol{u}}_{2}\right)\left(\widehat{\boldsymbol{w}}_{1} \cdot \widehat{\boldsymbol{u}}_{2}\right)\right]
$$

which is identical with the expression in Equation (A11) except for the sign of the imaginary term. Thus, because of the symmetry considerations delineated in Section 2 it seems sensible to introduce

$$
\mathcal{S}_{220}^{20}+\mathcal{S}_{220}^{20}=\sqrt{\frac{3}{10}} \mathbf{B}_{1}: \mathbf{U}_{2} .
$$

Finally, repeating the above analysis with $L_{2 \pm}$ instead of $L_{1 \pm}$ we would finally arrive at

$$
\mathcal{S}_{220}^{02}+\mathcal{S}_{220}^{02}=\sqrt{\frac{3}{10}} \mathbf{U}_{1}: \mathbf{B}_{2}
$$

and thus the coupling between uniaxial and biaxial degrees of freedom to $u_{\text {aniso }}$ would be proportional to $\mathbf{B}_{1}: \mathbf{U}_{2}+\mathbf{U}_{1}: \mathbf{B}_{2}$. However, in the interest of minimum complexity of the model we shall follow Sonnet et al. [5] and disregard this type of coupling altogether. This last expression can be obtained by symmetry arguments. Because the 
mesogens are identical we can interchange the labels 1 and 2 and replace $n_{1}$ by $n_{2}$ and vice versa.

Finally, using the same calculus as above and successively applying $L_{2 \pm}$ twice to Equations (A11) and (A12) we obtain four equations. For symmetry reasons discussed in Section 2 we can use Equation (9). One notices that each term on the right-hand side of this latter expression is of the general form

$$
\frac{1}{4} \sqrt{\frac{1}{5}}\left[\left(\widehat{\boldsymbol{v}}_{1} \mp i \widehat{\boldsymbol{w}}_{1}\right) \cdot\left(\widehat{\boldsymbol{v}}_{2} \mp i \widehat{\boldsymbol{w}}_{2}\right)\right]^{2},
$$

where the plus sign applies if the corresponding integer of $\mathcal{S}_{220}^{n_{1} n_{2}}$ on the right-hand side of Equation (9) is negative and vice versa. Using the definition of the biaxial tensor $B$ given in Equation (10b) as well as Equation (B.16) of Ref. 38 we obtain

$$
\mathcal{S}_{220}^{ \pm 2 \pm 2}=\sqrt{\frac{1}{5}} \mathbf{B}_{1}: \mathbf{B}_{2}
$$

as our final result.

\section{Appendix B. Limiting value of the orientation correlation function}

In this Appendix we rationalise Equations (33) for the limiting $\left(r_{12} \rightarrow \infty\right)$ behaviour of the orientation correlation functions introduced in Equations (26a) and (26b). Using Equations (30) and (31) we have

$$
\begin{aligned}
\left\langle\mathcal{S}_{l l 0}^{n_{1} n_{2}}\right\rangle_{\infty}= & \frac{(-)^{l}}{\sqrt{2 l+1}}\left(\frac{2 l+1}{8 \pi^{2}}\right)^{2} \sum_{m}(-)^{m} \\
& \times \sum_{l_{1} m_{1} k_{1}} s_{\left|m_{1}\right|\left|k_{1}\right|} \alpha_{\left|m_{1}\right|\left|k_{1}\right|}^{l_{1}} \\
& \times \int \mathrm{d} \Omega_{1} \mathcal{D}_{m_{1} k_{1}}^{l_{1}}\left(\Omega_{1}\right) \mathcal{D}_{\underline{m n}_{1}}^{l *}\left(\Omega_{2}\right) \\
& \times \sum_{l_{2} m_{2} k_{2}} s_{\left|m_{2}\right|\left|k_{2}\right|} \alpha_{\left|m_{2}\right|\left|k_{2}\right|}^{l_{2}} \\
& \times \int \mathrm{d} \Omega_{2} \mathcal{D}_{m_{2} k_{2}}^{l_{2}}\left(\Omega_{2}\right) \mathcal{D}_{m \underline{n}_{2}}^{l *}\left(\Omega_{2}\right),
\end{aligned}
$$

where the extra factors in the summations over $l_{1,2}, m_{1,2}$ and $k_{1,2}$ are introduced for the same reason as in Equation (18). Using Equation (A.93) of Ref. 38 this can be rewritten as

$$
\begin{aligned}
\left\langle\mathcal{S}_{l l 0}^{n_{1} n_{2}}\right\rangle_{\infty}= & \frac{(-)^{l}}{\sqrt{2 l+1}} \sum_{m}(-)^{m} \sum_{l_{1} m_{1} k_{1}} \sum_{l_{2} m_{2} k_{2}} s_{\left|m_{1}\right|\left|k_{1}\right|} s_{\left|m_{2}\right|\left|k_{2}\right|} \\
& \times \alpha_{\left|m_{1} \| k_{1}\right|}^{l_{1}} \alpha_{\left|m_{2} \| k_{2}\right|}^{l_{2}} \delta_{l l_{1}} \delta_{l l_{2}} \delta_{\underline{n}_{1}} k_{1} \delta_{\underline{n}_{2} k_{2}} \delta_{m_{1} \underline{\underline{m}}} \delta_{m_{2} m} .
\end{aligned}
$$


We restrict the evaluation of this expression to even $l$ for reasons already stated in Section 2. In addition, the Kronecker symbols cause several of the summations to collapse so that we can rewrite the previous equation more compactly as

$$
\begin{aligned}
\sqrt{2 l+1}\left\langle\mathcal{S}_{l l 0}^{n_{1} n_{2}}\right\rangle_{\infty}= & \sum_{m_{1} m_{2} m}(-)^{m} s_{\left|m_{1}\right|\left|n_{1}\right|} s_{\left|m_{2}\right|\left|n_{2}\right|} \\
& \times \alpha_{\left|m_{1}\right|\left|n_{1}\right|}^{l} \alpha_{\left|m_{2}\right|\left|n_{2}\right|}^{l} \delta_{m_{1} \underline{\underline{m}}} \delta_{m_{2} m} .
\end{aligned}
$$

Noticing that the integers $m_{1}$ and $m_{2}$ have to be even on account of the symmetry of the odf [21] and evaluating the last two Kronecker symbols in the previous expression we obtain

$$
\sqrt{2 l+1}\left\langle\mathcal{S}_{l l 0}^{n_{1} n_{2}}\right\rangle_{\infty}=\sum_{m=0}^{l} \alpha_{m\left|n_{1}\right|}^{l} \alpha_{m\left|n_{2}\right|}^{l} 2^{\delta_{n_{1} 0}+\delta_{n_{2} 0}-2},
$$

where we evaluated $s_{\left|m_{1}\right|\left|k_{1}\right|} s_{\left|m_{2}\right|\left|k_{2}\right|}$ using Equation (32). Notice that in Equation (B4), the summation is carried out only for positive $m$ because terms for positive and negative values of $m$ are identical. This brings in an extra factor of 2 except for $m=0$. To correct for this we have introduced $2^{-\delta_{m 0}}$. In addition, it should be borne in mind that on

account of the symmetry of the odf, $\alpha_{\left|m_{1}\right|\left|k_{1}\right|}^{l}$ and $\alpha_{\left|m_{2}\right|\left|k_{2}\right|}^{l}$ vanish for odd values of $m_{1}$, $m_{2}, k_{1}$, or $k_{2}$ from the outset. From Equation (B4) it is easy to derive Equations (33) and (34).

\section{References}

[1] P.G. de Gennes and J. Prost, The physics of liquid crystals, 2nd ed. (Oxford University Press, Oxford, 1995).

[2] M.J. Freiser, Phys. Rev. Lett. 24, 1041-1043 (1970).

[3] R. Alben, J. Chem. Phys. 59, 4299-4304 (1973).

[4] J.H. Lee, T.K. Lim, W.T. Kim and J.I. Jin, J. Appl. Phy. 101, 034105 (2007).

[5] A.M. Sonnet, E.G. Virga and G.E. Durand, Phys. Rev. E 67, 061701 (2003).

[6] J.C. Eichler, R.A. Skutnik, A. Sengupta, M.G. Mazza and M. Schoen, Mol. Phys. 117, 3715-3733 (2019).

[7] R.A. Skutnik, L. Lehmann, S. Püschel-Schlotthauer, G. Jackson and M. Schoen, Mol. Phys. 117, 2830-2845 (2019).

[8] A. Cuetos, A. Galindo and G. Jackson, Phys. Rev. Lett. 101, 237802 (2008).

[9] L.J. Yu and A. Saupe, Phys. Rev. Lett. 45, 1000-1003 (1980).

[10] E.A. Oliveira, L. Liebert and A.M. Figueiredo Neto, Liquid Crystals 5, 1669-1675 (1989).

[11] A. Stroobants and H.N.W. Lekkerkerker, J. Phys. Chem. 88, 3669-3674 (1984).

[12] H.H. Wensink, G.J. Vroege and H.N.W. Lekkerkerker, Phys. Rev. E 66, 041704 (2002).

[13] R.Y. Dong, S. Kumar, V. Prasad and J. Zhang, Chem. Phys. Lett. 448, 54-60 (2007).

[14] M. Lehmann, C. Köhn, J.L. Figueirinhas, G. Feio, C. Cruz and R.Y. Dong, Chem. Eur. J. 16, 8275-8279 (2010).

[15] B.R. Acharya, A. Primak and S. Kumar, Liquid Crystals Today 13, 1-4 (2004).

[16] E. van den Pol, A.V. Petukhov, D.M.E. Thies-Weesie, D.V. Byelov and G.J. Vroege, Phys. Rev. Lett. 103, 258301 (2009).

[17] C. Cruz, J.L. Figueirinhas, D. Filip, G. Feio, A.C. Ribeiro, Y. Frère, T. Meyer and G.H. Mehl, Phys. Rev. E 78, 051702 (2008).

[18] A. Eremin, S. Diele, G. Pelzl, H. Nádasi, W. Weissflog, J. Salfetnikova and H. Kresse, Phys. Rev. E 64, 051707 (2001). 
[19] D.Y. Kim, L. Wang, Y. Cao, X. Yu, S.Z.D. Cheng, S.W. Kuo, D.H. Song, S.H. Lee, M.H. Lee and K.U. Jeong, J. Mater. Chem. 22, 16382-16389 (2012).

[20] B. Tjipto-Margo and G.T. Evans, J. Chem. Phys. 94, 4546-4556 (1991).

[21] B.M. Mulder, Liq. Cryst. 1, 539-551 (1986).

[22] M.P. Allen, Liq. Cryst. 8, 499-511 (1990).

[23] P.J. Camp and M.P. Allen, J. Chem. Phys. 106, 6681-6688 (1998).

[24] J.P. Straley, Phys. Rev. A 10, 1881 (1974).

[25] R. Berardi, C. Fava and C. Zannoni, Chem. Phys. Lett. 236, 462-468 (1995).

[26] R. Berardi and C. Zannoni, J. Chem. Phys. 113, 5971-5979 (2000).

[27] F. Biscarini, C. Chiccoli, P. Pasini, F. Semeria and C. Zannoni, Phys. Rev. Lett. 75, 1803 (1995).

[28] A. Cuetos, M. Dennison, A. Masters and A. Patti, Soft Matter 13, 4720-4732 (2017).

[29] A.J. Stone, in The molecular physics of liquid crystals, edited by G. R. Luckhurst and G. W. Gray (Academic Press, London, 1979), Chap. 2, pp. 31-50.

[30] G.R. Luckhurst and S. Romano, Mol. Phys. 40, 129-139 (1980).

[31] L. Longa and G. Pająk, Liq. Cryst. 32, 1409-1417 (2005).

[32] L. Longa, P. Grzybowski, S. Romano and E. Virga, Phys. Rev. E 71, 051714 (2005).

[33] Y. Martínez-Ratón, S. Varga and E. Velasco, Phys. Chem. Chem. Phys. 13, 13247-13254 (2011).

[34] A.J. Stone, Mol. Phys. 36, 241-256 (1978).

[35] A. Messiah, Quantum mechanics, Vol. 2 (North Holland, Amsterdam, 1969).

[36] A.M. Sonnet and E.G. Virga, Dissipative ordered fluids: theories for liquid crystals (Springer Science \& Business Media, New York, 2012).

[37] C. Zannoni, in The molecular physics of liquid crystals, edited by G. R. Luckhurst and G. W. Gray (Academic Press, London, 1979), Chap. 3, pp. 51-83.

[38] C.G. Gray and K.E. Gubbins, Theory of molecular fluids, Vol. 1 (Clarendon Press, Oxford, 1984).

[39] D.M. Brink and G.R. Satchler, Angular momentum, 2nd ed. (Clarendon Press, Oxford, 1968), pp. 146-148.

[40] A.A. Albert and B. Muckenhoupt, Mich. Math. J. 4, 1-3 (1957).

[41] W. Maier and A. Saupe, Z. Naturforsch. A 15, 287-292 (1960).

[42] M. Schoen, A.J. Haslam and G. Jackson, Langmuir 33, 11345-11365 (2017).

[43] C. Zannoni, in The molecular physics of liquid crystals, edited by G. R. Luckhurst and G. W. Gray (Academic Press, London, 1979), Chap. 9, pp. 191-220.

[44] W.B. Streett and D. Tildesley, Proc. Roy. Soc. (London) A 348, 485-510 (1976).

[45] R. Eppenga and D. Frenkel, Mol. Phys. 52, 1303-1334 (1984).

[46] M.P. Allen and D.J. Tildesley, Computer simulation of liquids, 2nd ed. (Oxford university press, Oxford, 2017).

[47] D. Frenkel and B. Smit, Understanding molecular simulation: from algorithms to applications, Vol. 1, 2nd ed. (Academic Press, San Diego, 2001).

[48] B.S. John and F.A. Escobedo, J. Phys. Chem. B 109, 23008-23015 (2005).

[49] B.S. John, C. Juhlin and F.A. Escobedo, J. Chem. Phys. 128, 044909 (2008).

[50] A. Cuetos, E.M. Rafael, D. Corbett and A. Patti, Soft Matter 15, 1922-1926 (2019).

[51] S. Schlotthauer, R.A. Skutnik, T. Stieger and M. Schoen, J. Chem. Phys. 142, 194704 (2015).

[52] S. Püschel-Schlotthauer, T. Stieger, M. Melle, M.G. Mazza and M. Schoen, Soft Matter 12, 469-480 (2016).

[53] S. Püschel-Schlotthauer, V.M. Turrión, T. T. Stieger, R. Grotjahn, C. Hall, M.G. Mazza and M. Schoen, J. Chem. Phys. 145, 164903 (2016).

[54] M. Schoen and C. Hoheisel, Mol. Phys. 57, 65-79 (1986).

[55] L. Blum and A.J. Torruella, J. Chem. Phys. 56, 303-310 (1972).

[56] L.A. Madsen, T.J. Dingemans, M. Nakata and E.T. Samulski, Phys. Rev. Lett. 92, 145505 (2004). 